Eur. J. Pers. 16: 1-41 (2002)

DOI: $10.1002 /$ per.431

\title{
From Adorable to Worthless: Implicit and Self-Report Structure of Highly Evaluative Personality Descriptors
}

\author{
VERÓNICA BENET-MARTÍNEZ ${ }^{1 *}$ and NIELS G. WALLER ${ }^{2}$ \\ ${ }^{1}$ University of Michigan, Ann Arbor, MI, USA \\ ${ }^{2}$ Vanderbilt University, Nashville, Tennessee, USA
}

\begin{abstract}
So-called highly 'evaluative' personality judgments (e.g. describing someone as exceptional,odd, or vile,) are an integral component of people's daily judgments of themselves and others. However, little is known about the conceptual structure, psychological function, and personality-relevance of these kinds of attribution. Two studies were conducted to explore the internal (i.e. implicit) and external (i.e. self-report) structure of highly evaluative terms. Factor analyses of semantic-similarity sortings and self-reports on several representative samples of highly evaluative personality adjectives yielded internal and external structures that were very similar. Both types of structure included five dimensions representing distinction, worthlessness, depravity, unconventionality, and stupidity. The robustness of the uncovered dimensions across the two studies suggests that typically excluded highly evaluative personality terms, far from being behaviorally ambiguous and psychologically uninformative, allude to meaningful dispositions that people both implicitly understand and possess to different degrees. These findings also suggest that highly evaluative personality judgments are organized around the basic domains of morality (i.e. depravity), power (distinction and worthlessness), peculiarity (unconventionality), and intelligence (stupidity). We discuss the implications of our findings for the study of self- and other-esteem processes, personality perception, and the Big Seven factor model of personality. Copyright (C) 2002 John Wiley \& Sons, Ltd.
\end{abstract}

\section{INTRODUCTION}

Evaluation is perhaps the single most important concept associated with feeling, thinking, and doing (Tesser and Martin, 1996, p. 400).

\footnotetext{
*Correspondence to: Verónica Benet-Martínez, Department of Psychology, 3251 East Hall, University of Michigan, Ann Arbor, MI 48109, USA. E-mail: veronica@umich.edu.

Contract/grant sponsor: University of Michigan's Rackham School of Graduate Studies.

Contract/grant sponsor: Spanish Ministry of Science and Educación (Ministerio de Educatión y Ciencia).
} 
The social function and ubiquity of evaluative (versus denotative or descriptive) person ascriptions in daily discourse has been recognized in linguistics (Maalej, unpublished; manuscript; Rivière, 1983; Simon-Vandenbergen, 1995), psychology (Kaplan, 1975; Lewicka, 1979), and philosophy (Blumberg, 1973). Social cognitive research, for instance, shows that highly evaluative personality ascriptions (e.g., describing someone as excellent, immoral, strange, or conventional) play a unique role in person perception processes (Wojciszke, Brycz and Borkenau, 1993). Specifically, highly evaluative personality judgments facilitate future person inferences by eliciting affect-based expectations about the kinds of trait and behavior that characterize a person (Stephens, unpublished dissertation; Van der Pligt and Taylor, 1984). The unique social function played by evaluative person judgments is also supported by developmental linguistics research showing that even young children rely on largely evaluative (versus descriptive) statements when talking about themselves and others (Burger and Miller, 1999; Hoff-Ginsberg, 1997). Furthermore, evidence from cognitive psychology indicates that evaluative judgments frequently dominate over narration and description in informal communication (Giammatteo, 1998) and conversational recall (Hyman, 1994).

The role of evaluation in person ascriptions has also been acknowledged explicitly by some personality psychologists (Borkenau, 1990; Bromley, 1977; Buss, 1991; Hogan, 1982, 1996; Tellegen, 1993; Waller, 1999). For instance, recent work on the sociocognitive function of trait usage suggests that personality terms '.. that are clearly desirable or undesirable are more numerous than terms that are neutral in evaluation. This is a reasonable finding if the prime purpose of trait terms is to inform about the aptitude of persons' (p. 394, italics added, Borkenau, 1990). Similarly, taking a socio-evolutionary perspective, Hogan (1982) asserts that the main purpose of personality ascriptions is to evaluate the potential of persons as resources for the group. Evaluation is also seen as a core element in important personality processes such as self-esteem and self-concept (O'Brien and Epstein, 1988; Roid and Fitts, 1988), self-perceptual biases (Paulhus and John, 1998; Rosenberg and Sedlak, 1972; Wiggins, 1964), and even personality disorders (Tellegen, 1993; Waller, 1999).

The view that highly evaluative self- and other-judgments are an intrinsic element of person perception has not permeated the personality taxonomy literature, where popular structural models, like the Big Five (or Five Factor Model), define the personality domain primarily in terms of descriptive traits. ${ }^{1}$ Historically, this emphasis on descriptive meaning (over evaluation) when mapping the personality domain can be traced to traditional definitions of personality that dismiss 'moral judgments' and reputations as not relevant to the scientific study of personality (Allport and Odbert, 1936; Cattell, 1943; Norman, 1967; but also see Tellegen, 1993; Waller, 1999). The exclusionary argument held by these definitions is at odds with previously introduced contemporary socio-cognitive and

\footnotetext{
${ }^{1}$ The common distinction in the literature between evaluative and descriptive judgments is useful here given the purposes of our discussion and the work presented. This distinction, however, is artificial because, in reality, descriptive and evaluative meaning are not discrete categories but a continuum; that is, most personality descriptors have both a descriptive and an evaluative component, although to a different extent (Di Blas, Forzi and Peabody, 2000; Jauregui, 1973; Marquez, 1998). Consider for instance the words assertive and aggressive. Notice that these words share a descriptive component (social forcefulness) but vary in their evaluative component (aggressive is more evaluative than assertive). Given this, and to distinguish the extreme terms that are the focus of this research (e.g. lousy) from the more descriptive (but also evaluative) terms that are traditionally used in personality studies, we use the term 'highly evaluative' personality descriptor throughout our paper to refer to the stimuli used in our studies.
} 
evolutionary perspectives, which see evaluative judgments as particularly informative regarding dispositions and behaviors of highly affective relevance to humans. That is, evaluative judgments are informative about social characteristics that are relevant to group survival (Borkenau, 1990). The historical disregard for highly evaluative personality terms is also challenged by recent lexical personality work (Benet-Martínez and Waller, 1997; Tellegen and Waller, 1987; Waller, 1999). This literature indicates that the traditional lexical personality space can be expanded with two additional dimensions tapping different aspects of evaluation, a model known as the 'Big Seven' factor structure (Tellegen and Waller, 1987).

Two interesting issues derive from the aforementioned ideas. First, if evaluative personality judgments are particularly informative about what is important to humans, then exploring the number and nature of the basic conceptual dimensions underlying highly evaluative personality terms becomes an important endeavour. Second, to the extent that traditional lexical models of personality have systematically excluded highly evaluative terms, the resulting dimensions may be informative regarding personality domains possibly unrepresented in traditional personality studies.

These issues guided the two studies presented in this paper. The main goal of our two studies is to conduct an initial examination of the structure underlying highly evaluative personality adjectives, more specifically (a) to identify and compare the implicit (internal) and self-report (external) dimensions obtained from semantic-similarity sortings and selfreports collected on a large and representative pool of highly evaluative personality adjectives and (b) to discuss the implications of our findings for the study of the descriptive and evaluative components of personality description, and the Big Seven factor model (Tellegen and Waller, 1987). We see our exploration of the dimensions underlying the highly evaluative personality lexicon as the initial phase of a larger research programme on the evaluative domain of personality description (Benet-Martínez, manuscript in preparation).

Before turning to our studies, we first discuss the difference between implicit and selfreport dimensions and review the evidence for the Big Seven's evaluative personality dimensions. We finish our introduction with a brief account of some empirical attempts at separating the descriptive and evaluative components of personality.

\section{Implicit and self-report structures}

Psychologists have developed dimensional models to organize variation in a wide range of psychological processes such as personality traits (Allport and Odbert, 1936; Cattell, 1943; Goldberg, 1982; John, 1990; McCrae and Costa, 1985; Tellegen and Waller, in press), emotions (Russell and Barrett, 1999; Watson, Wiese, Vaidya and Tellegen, 1999), and motives (Emmons, 1997; Maslow, 1970; Pervin, 1983). Empirical approaches to this issue typically have relied on two methods: (a) covariance structure analysis of selfor other-report data, and (b) structural analysis of semantic-similarity judgments. Dimensions obtained from the first approach reflect external structures because they arise from the co-occurrences of psychological attributes within individuals (Wiggins, 1973). Positive and Negative Affect are two dimensions representative of this approach (Watson, Clark and Tellegen, 1988). Dimensions obtained from structural analysis of semantic similarity data are known as internal structures because they putatively reflect people's implicit (i.e. cognitive) theories about the conceptual relations among attributes (Wiggins, 1973). The Intimacy, Passion, and Commitment dimensions that emerge from 
implicit theories of love are representative of this second tradition (Hassebrauck and Thomas, 1996; Sternberg, 1988).

An important finding from the literature on dimensional models of personality is that the Big Five (Goldberg, 1993), also known as the Five Factor Model (McCrae and Costa, 1997), emerges not only from self-reports and ratings of well known others and strangers (Mervielde, 1994; Peabody and Goldberg, 1989), but also from semantic similarity ratings and co-occurrence likelihood ratings (Borkenau, 1992). This unexpected finding (that the Big Five can be reliably identified in self- and observer-report data as well as in semantic similarity data) spurred a debate during the 1970s and 1980s regarding the conceptual status and distinguishing features of external and internal personality structures. ${ }^{2}$ Some researchers argued that external structures do not reflect the actual relations among personality attributes but merely denote people's folk beliefs about the semantic/ conceptual relations among personality-descriptive terms (the 'systematic distortion' hypothesis; Shweder and D'Andrade, 1980). These researchers concluded that external structures are inaccurate models of how we see ourselves or others. Others researchers argued that the resemblance between dimensions obtained from trait ratings and similarity judgments was to be expected because internal structures are acquired through everyday observation of people's trait descriptive behaviour (the 'accurate reflection' hypothesis; Block, Weiss and Thorne, 1979). ${ }^{3}$

A third position, now widely accepted and known as the 'overlap' hypothesis (Borkenau, 1992), posits that external structures reflect both implicit personality theories (internal structures) and the actual covariation among traits. This so-called 'overlap model' expands the 'accurate reflection' model by stating that implicit personality theories reflect actual behavioural co-occurrences (by virtue of being largely acquired through experience) as well as cultural models (D'Andrade, 1985) and evolutionary forces (Buss, 1991; Hogan, 1982). According to this later view, the Big Five represents a basic structure of individual differences and individuals' implicit beliefs about personality (Borkenau, 1992; John and Robins, 1993; Sneed, McCrae and Funder, 1998).

\section{The Big Seven factor models of personality}

Despite the widespread acceptance of the Big Five among personality researchers, recent work (Paunonen and Jackson, 2000; Tellegen, 1993; Waller, 1999) has suggested the need to provide a broader scope of the personality domain by considering dimensions outside the Big Five. The view of Tellegen and Waller in particular, is based on the previously introduced fact that the Big Five developed from a pool of personality-relevant terms that excluded highly evaluative terms (and many state-mood descriptors) because these descriptors were not considered representative of so called biophysical traits (Allport and Odbert, 1936; Cattell, 1943; Norman, 1967; see John, Angleitner and Ostendorf, 1988, for an excellent review of all these studies).

\footnotetext{
${ }^{2}$ It should be noted that in the 1989 study of Peabody and Goldberg, external and internal personality structures were quite similar, but the dimensions derived from semantic sortings were generally simpler, more schematic, and accounted for more variance. Peabody and Goldberg explained these differences in terms of the cognitive economy associated with semantic judgments.

${ }^{3}$ See Larsen and Diener (1992) and Reisenzein and Schimmack (1999) for a discussion of similar debate in the emotion domain.
} 
To examine the consequences of the above exclusionary criteria, Tellegen and Waller (1987) re-examined the English personality lexicon. Unlike their predecessors, these researchers avoided a priori exclusion criteria for highly evaluative and state descriptors when selecting personality terms from the dictionary and, interestingly, they identified seven (rather than five) higher-order personality dimensions. 'Their so-called 'Big Seven' structure differs from earlier taxonomies in that it includes two clearly evaluative dimensions labeled Positive and Negative Valence. These Valence dimensions are characterized by terms such as: excellent, special, impressive, versus ordinary, average, unexceptional (Positive Valence), and wicked, awful, disgusting, immoral (Negative Valence). The other five dimensions (Positive Emotionality, Negative Emotionality, Conscientiousness, Agreeableness, and Conventionality) are closely related to the Big Five's dimensions of Extroversion, Neuroticism, Conscientiousness, Agreeableness, and Openness respectively. ${ }^{5}$

Importantly, the non-restrictive criteria of Tellegen and Waller (1987) for descriptor selection also allowed emotion-laden descriptors (e.g. peppy, spirited, or jumpy, jittery) to emerge in the Big Seven factor structure. These affect terms, in conjunction with traditional markers of Extraversion and Neuroticism, formed two independent dimensions which Tellegen and Waller called Positive and Negative Emotionality, rather than Extraversion and Neuroticism, to reflect their emotional core.

The use of non-restrictive selection criteria broadened the resulting personality structure in at least one more way. Specifically, it allowed evaluative terms denoting extreme openness (e.g. peculiar, odd, unusual) to emerge in the negative pole of the Big Seven's Conventionality dimension, next to other terms denoting progressive political-social attitudes and intellectual curiosity (i.e. Openness to Experience).

During the last decade, several replications of the Big Seven have been obtained across samples, targets, and languages (Almagor, Tellegen and Waller, 1995; Benet and Waller, 1995; Benet-Martínez and Waller, 1996; Waller, 1999). ${ }^{6}$ Factors resembling Negative and (to a lesser extent) Positive Valence were also found by Saucier (1997) in a study that examined the effect of personality variable selection on the structure of personality ratings. A Negative Valence factor has also been found in lexical studies of Tagalog and Dutch personality descriptors (Church, Reyes, Katigbak and Grimm, 1997; Church, Katigbak and Reyes, 1998; De Raad and Hoskens, 1990; De Raad, Mulder, Kloosterman and Hofstee, 1988).

Regarding the validity of the Big Seven Valence dimensions, we believe that these constructs relate to components of self-evaluation or esteem that are not explicitly represented in previous lexical models (Benet-Martínez, 1999; Tellegen, 1993; Waller, 1999). Specifically, Positive and Negative Valence capture reliable variance in selfperceptions of status and prominence (Positive Valence) and virtue (Negative Valence), what Paulhus and John (1998) describe as egoistic and moralistic self-perceptual biases, or the 'superhero' and 'sainthood' complexes (see also Rosenberg and Sedlak, 1972; Wiggins, 1964). The psychological significance of Positive and Negative Valence is also supported by some multidimensional models of self-esteem and self-concept (O'Brien and

\footnotetext{
${ }^{4}$ See Waller (1999) for a detailed description of the stratified-sampling procedures used for the selection of personality terms in the 1987 study of Tellegen and Waller.

${ }^{5}$ For labels and examples of marker items for each of the Big Seven dimensions, see Table 1 in Benet and Waller (1995).

${ }^{6}$ The 1995 study of Almagor et al. provides only a partial replication of the Big Seven given that a dimension representing Conventionality failed to emerge.
} 
Epstein, 1988; Roid and Fitts, 1988) that include dimensions representing 'personal power' and 'moral self-approval,' two constructs that can be conceptually linked to Positive and Negative Valence, respectively. ${ }^{7}$

Lastly, Positive and Negative Valence might also prove useful for understanding of Axis II personality disorders (Waller, 1999). Specifically, these dimensions may help elucidate the maladaptive self-evaluative processes involved in narcissism (which is characterized by a grandiose sense of self-importance), borderline personality (which involves alternation of extreme overidealization and devaluation of the self) or avoidant personality (which involves social discomfort and fear of negative evaluation by others).

Some researchers have argued that Positive and Negative Valence do not capture meaningful personality variance outside the Big Five, but rather merely represent extreme variants of traits located within the five-factor model (McCrae and Costa, 1995; Saucier, 1994a). Only a handful of studies have empirically addressed this issue. McCrae and Costa (1995) conducted a joint exploratory factor analysis of Big Five and Big Seven scales and reported a five-factor solution where Positive Valence loaded significantly on Extraversion and Openness, and Negative Valence loaded on Neuroticism, Extraversion, Conscientiousness, and Agreeableness. The authors interpreted these results as evidence that the two evaluative dimensions do not carry enough substantive variance beyond the Big Five. Results from recent correlational and confirmatory factor analytic studies of the Big Five/ Big Seven independence, however, contradict this conclusion (Benet-Martínez, 1999; Benet-Martínez and Waller, 1997). These studies show that Positive and Negative Valence scales have small to non-significant correlations with Big Five scales (see Table 1 in Benet-Martínez and Waller, 1997), and that the latent structure underlying Big Seven and Big Five measures is best organized around seven dimensions representing the Big Five plus Positive and Negative Valence (see CFA figure in Benet-Martínez, 1999), supporting the view that Positive and Negative Valence may capture sufficient unique psychological variance to define factors outside the Big Five. ${ }^{8}$

\section{Descriptive and evaluative components of personality}

The Big Five do not include dimensions representing positive and negative evaluation but this does not imply that Big Five personality descriptors are devoid of evaluation. As stated previously, the typical personality descriptor has both a descriptive and an evaluative component, although to a different extent. In fact, several personality researchers have shown that structures separating description and evaluation can be obtained from Big Five descriptors (Di Blas et al., 2000; Peabody, 1984; Saucier, 1994b) or other descriptor lists where trait words are over-represented (Kuusinen, 1969; Kim and Rosenberg, 1980; Osgood and Suci, 1955), even though in many instances these descriptors were selected a priori for their low evaluativeness (Allport and Odbert, 1936; Norman, 1967). Peabody

\footnotetext{
${ }^{7}$ The psychological significance of Positive and Negative Valence and their orthogonality may be linked to basic evaluative processes similar to those involved in the attitude domain (Cacioppo, Gardner and Berntson, 1997). Recent evidence from this area suggests that, contrary to traditional bipolar conceptualizations, different motivational systems underlie judgments of the positive and negative significance of a stimulus (Cacioppo et al., 1997; Tesser and Martin, 1996).

${ }^{8}$ Evidence also exists indicating that Positive and Negative Valence are not 'difficulty' factors (Waller, 1999) and are only weakly related to measures of social desirability and acquiescence (McCrae and Costa, 1995). A recent self-peer convergence study also suggests that these two dimensions capture observable meaningful personality variance that cannot be fully accounted by the Big Five (Benet-Martinez and Waller, 1996).
} 
(1984; Peabody and Goldberg, 1989), for instance, identified two descriptive dimensions (tight versus loose and assertive versus unassertive) and one general (i.e. correlated with the other two) positive versus negative evaluative factor. Using Big Five descriptors, Saucier (1994b) identified five dimensions, of which four were descriptive (extraversion, affective orientation, norm orientation, and form orientation) and one evaluative. Similarly, Osgood and Suci (1955), using personality scales and other terms assembled by various methods, identified dimensions representing evaluation, activity, and potency.

The literature reviewed so far supports the view that personality descriptors (including those that have been selected a priori for their low evaluativeness, such as those that typically define the Big Five) have robust and identifiable underlying evaluative components. This conclusion raises an intriguing question: can the same logic be applied to traditionally excluded highly evaluative personality descriptors? That is, does this descriptor class have reliable personality-relevant descriptive components? Work discussed earlier (Benet-Martínez, 1999; Tellegen, 1993; Tellegen and Waller, 1987), as well as recent studies conducted by Big Five researchers (Saucier, 1997; Saucier and Goldberg, 1998), suggest that the answer to these questions may be yes. These studies show that when examined together with less-evaluative, traditional Big Five markers, highly evaluative descriptors define two independent dimensions representing depravity versus morality (Negative Valence) and social potency or power versus inferiority (Positive Valence) that are not subsumable within the Big Five (although the evidence for the Big Five independence of Positive Valence is less clear in the studies of Saucier and Goldberg).

An important question remains unanswered with regard to the conceptual and psychometric status of these additional evaluative personality dimensions. Namely, insofar as the emergence and meaning of a particular dimension is heavily influenced by the diversity of stimuli included in the structural analysis, what would be the number and nature of dimensions emerging from pools of highly evaluative personality terms that $d o$ not explicitly include Big Five adjectives? More specifically, would these conceptual dimensions look just like Positive and Negative Valence or represent instead narrower, more specific subcomponents of these two broad constructs? Alternatively, would these uncovered dimensions perhaps capture domains of evaluation never uncovered previously?

A secondary issue concerns the degree of overlap between the external and internal structures underyling highly evaluative personality terms. That is, would people's implicit theories about the conceptual relations among highly evaluative personality descriptors correspond closely to the actual covariation in people of the dispositions represented by these terms? Note that this last question, which has received large theoretical and empirical attention in the emotion and trait domain, has yet to be addressed in the domain of evaluation. The finding that external and internal structures for highly evaluative personality terms do in fact overlap would suggest that, as with traits and emotions (Borkenau, 1992; Schimmack and Reisenzein, 1997), evaluative personality terms allude to psychological dispositions of socio-evolutionary relevance that people both implicitly understand and possess to different degrees.

The two studies presented next provide an initial examination of the issues discussed so far. These studies identify and compare the internal and external structures underlying semantic-similarity sortings and self-reports on a large and representative pool of highly evaluative personality adjectives. 


\title{
STUDY 1: INTERNAL STRUCTURE OF HIGHLY EVALUATIVE PERSONALITY DESCRIPTORS
}

\section{Method}

Derivation of three samples of highly evaluative personality terms

Three representative samples of highly evaluative personality adjectives (each sample included 75 terms) were randomly selected from Norman's (1967) excluded category of 'evaluative' terms. We selected only a subset of Norman's original set of 760 evaluative descriptors (using a random-number generator) because this list was too large to be used in a single meaning-similarity exercise. The three samples were highly independent from each other such that only a handful of terms overlapped among the three sets (eight common terms appeared in sets 1 and 2; six common terms appeared in sets 1 and 3; five common terms appeared in sets 2 and 3; and two common terms appeared in sets 1, 2, and 3).

The three sets of adjectives were used in separate free sorting exercises, an efficient and widely used method for obtaining judgments of meaning similarity (Brewer and Lui, 1996). Each term was printed on a $3 \times 5$ index card. Because many of the terms were potentially unfamiliar and/or ambiguous, each card also included an easy-to-understand short definition or synonym for the word (taken from various dictionaries). The complete list of 225 highly evaluative adjectives and their definitions is included in the Appendix.

\section{Sample}

Three samples of individuals sorted each set of terms (samples 1, 2, and 3 sorted sets 1, 2 and 3, respectively). All participants were undergraduate students at the University of California at Berkeley who received course credit for their participation. English was the first language of all participants. A wide range of college majors was represented, and the majority of participants were non-psychology students.

Sample 1 consisted of 41 individuals (15 men and 26 women) with a mean age of 20.13 years $(\mathrm{SD}=2.52)$. Sample 2 consisted of 48 individuals ( 23 men and 25 women) with a mean age of 20 years $(\mathrm{SD}=1.89)$. Sample 3 consisted of 41 individuals $(12$ men and 29 women) with a mean age of 21.46 years $(\mathrm{SD}=1.94)$.

\section{Procedure}

Each participant was seated next to a large table and given the following printed instructions:

\begin{abstract}
You will be presented with a set of 75 cards. Note that each card has a word in the middle and a definition or synonym for that word at the bottom. As you can see, all the words are evaluative and ordered alphabetically. Your job is to sort these words into groups that contain characteristics which are the same, or very similar. You can use as few or as many groups as you wish. Make the groups by making different piles on the table. Please first read each card very carefully, and think of its meaning as it applies to humans (not to objects). Very important: If you find words that are antonyms (i.e., words that have clear opposite meanings, such as 'brilliant' versus 'stupid'), put them in separate piles but, please, put the antonym piles right next to each other on the table (so we know that the piles represent opposite poles of the same group). When finished, check your piles to ensure that there are not groups that can be merged because they have very similar meanings.
\end{abstract}

After receiving these instructions, each participant performed the sorting task, finishing in an hour or less. The mean number of sorting groups used by participants in set 1 was 10 
$(\mathrm{SD}=5), 10.6$ in set $2(\mathrm{SD}=16)$, and 12.7 in set $3(\mathrm{SD}=5.6)$, counting antonym groups and their corresponding piles as a single group. Sortings from each participant were coded by assigning a unique integer to all terms sorted in the same pile (i.e. for each participant, as many different integers were assigned as number piles the participant had made). Antonym piles were assigned the same integer but of opposite sign (e.g. 3 and -3).

Using a computer program developed by Tellegen (unpublished, manuscript), the semantic sortings were converted to three (one per set) $75 \times 75$ co-occurrence matrices. The co-occurrence rate for a pair of terms was raised by one for each participant that assigned it to the same group. Each time a pair of terms was assigned to an antonym group the co-occurrence rate was decreased by one. This ensured that terms frequently placed in antonym piles by participants (e.g. unremarkable, distinctive) could receive a negative cooccurrence rate and that eventually both terms emerged as markers of the same evaluative dimension but with opposite-sign factor loadings. Because the number of participants sorting each set differed, the co-occurrence rates were converted to proportions by dividing each cell of the matrix by the number of participants. We then proceeded to investigate the major sources of variances (i.e. structure) for these three matrices using factor analysis. ${ }^{9}$

\section{Results}

\section{Factor analyses}

The goal of the factor analyses was to identify, if possible, a common dimensional structure that would best represent the internal (i.e. conceptual) structure of our highly evaluative personality adjectives within and across the three samples of terms. The application of factor analyses to sorting co-occurrence matrices has been used successfully by many personality researchers, including Church and Katigbak (1989), Harkness (1992), Watson et al. (1984), and Waller, Lykken and Tellegen (1995).

The first ten eigenvalues for set 1 were 16.1, 6.9, 6.1, 5.2, 3.9, 2.5, 2.5, 1.9, 1.7, and 1.4. For set 2 the first ten eigenvalues were 16.3, 8.6, 5.3, 4.8, 3.6, 2.7, 2.1, 1.9, 1.6, and 1.3; and for set 3 they were $12.3,8.7,6.3,4.9,3.3,2.9,2.4,2.0,1.8$, and 1.5. Using principal factors analysis, we first examined Varimax-rotated solutions with two to six factors. Furthermore, because structures including highly correlated components or a general evaluation factor plus additional content factors were also theoretically plausible (Osgood and Suci, 1955; Peabody, 1984), we also examined Promax- and Quartimax-rotated solutions. Selection of the final dimensional structures was guided by a mixture of quantitative and qualitative considerations: (1) the scree plots, (2) the psychological coherence of the obtained solutions, and (3) factor replicability within and across the three sets. We discuss the orthogonal solutions first.

Because presenting and describing in detail each of the Varimax-rotated structures (15 in total) goes beyond the space limitations of this paper, summaries of these solutions and examples of their primary markers are depicted in Tables 1 to 3. Note that the twofactor solutions for sets 1 and 3 included two very large factors broadly representing Positive (e.g. high-calibre, paragon) and Negative (scoundrel, cursed) Valence. The twofactor solution for set 2, however, was different in that it included one factor combining Positive and Negative Valence plus a factor representing Stupidity (e.g. noodle, blockhead). An interesting commonality among the three progressions of factor solutions

${ }^{9}$ Co-occurrence matrices are Gramian (can be represented by the product of a matrix and its transpose) and therefore can be factor analysed. 
Table 1. Semantic sortings of highly evaluative personality terms (set 1): varimax-rotated solutions and their highest factor markers

\begin{tabular}{|c|c|c|c|c|}
\hline 2 factors & 3 factors & 4 factors & 5 factors & 6 factors \\
\hline \multirow{24}{*}{$\begin{array}{l}\text { I } \\
\text { High-calibre } \\
\text { Demigod } \\
\text { Pluperfect } \\
\text { vs. } \\
\text { Pointless } \\
\text { Nonessential } \\
\text { Null } \\
(16.6 \%)\end{array}$} & \multirow{17}{*}{$\begin{array}{l}\text { I } \\
\text { High-calibre } \\
\text { Demigod } \\
\text { Excelled } \\
\text { vs. } \\
\text { Lunkhead } \\
\text { Blockhead } \\
\text { Gooney } \\
(15.3 \%)\end{array}$} & I & I & I \\
\hline & & Demigod & Pluperfect & Demigod \\
\hline & & Pluperfect & Demigod & Pluperfect \\
\hline & & High-calibre & Overgreat & Unflawed \\
\hline & & vs. & vs. & First-class \\
\hline & & Unideal & Unideal & Faultless \\
\hline & & Unexcellent & Unexcellent & High-calibre \\
\hline & & $\begin{array}{l}\text { Unexemplary } \\
(15.6 \%)\end{array}$ & $\begin{array}{l}\text { Unexemplary } \\
(15.3 \%)\end{array}$ & $(13.4 \%)$ \\
\hline & & & & $\begin{array}{l}\text { VI } \\
\text { Unexcellent } \\
\text { Unideal } \\
\text { Unexemplary } \\
\text { Third-class } \\
\text { Might-have-been } \\
\text { Inexperienced } \\
(5.5 \%)\end{array}$ \\
\hline & & $\downarrow$ IV & IV & \\
\hline & & Blockhead & Blockhead & Blockhead \\
\hline & & Blunderhead & Blunderhead & Blunderhead \\
\hline & & Lunkhead & Gooney & Gooney \\
\hline & & Half-witted & - Lunkhead & Lunkhead \\
\hline & & Gooney & Half-witted & Half-witted \\
\hline & & Assish & Assish & $\begin{array}{l}\text { Assish } \\
8.6 \%)\end{array}$ \\
\hline & & (10.8 & (10.0 & III \\
\hline & \multirow{7}{*}{$\begin{array}{l}\text { III } \\
\text { Nonessential } \\
\text { Unconsequential } \\
\text { Inessential } \\
\text { Pointless } \\
\text { Cipher } \\
\text { Unnecessary } \\
(10 \%)\end{array}$} & Nonessential & Nonessential & Nonessential \\
\hline & & Unconsequential & Unconsequential & Unconsequential \\
\hline & & Inessential & Inessential & Inessential \\
\hline & & Pointless & Pointless & Pointless \\
\hline & & Unnecessary & Unnecessary & Unnecessary \\
\hline & & Cipher & Cipher & Cipher \\
\hline & & $(9.4 \%)$ & $(9.4 \%)$ & $(9.2 \%)$ \\
\hline \multirow{18}{*}{$\begin{array}{l}\text { II } \\
\text { Welldoing } \\
\text { Adorable } \\
\text { Stainless } \\
\text { vs. } \\
\text { Bounder } \\
\text { Arrant } \\
\text { Scoundrel } \\
(14.4 \%)\end{array}$} & \multirow{18}{*}{$\begin{array}{l}\text { II } \\
\text { Welldoing } \\
\text { Adorable } \\
\text { Stainless } \\
\text { vs. } \\
\text { Bounder } \\
\text { Arrant } \\
\text { Scoundrel } \\
(13.5 \%)\end{array}$} & \multirow{18}{*}{$\begin{array}{l}\text { II } \\
\text { Welldoing } \\
\text { Adorable } \\
\text { Nifty } \\
\text { vs. } \\
\text { Bounder } \\
\text { Arrant } \\
\text { Scoundrel } \\
(12 \%)\end{array}$} & II & II \\
\hline & & & Welldoing & Welldoing \\
\hline & & & Adorable & Adorable \\
\hline & & & Nifty & Nifty \\
\hline & & & vs. & vs. \\
\hline & & & Bounder & Bounder \\
\hline & & & Arrant & Arrant \\
\hline & & & Scoundrel & Scoundrel \\
\hline & & & & $(I 2 \%)$ \\
\hline & & & $\mathrm{V}$ & $\mathrm{V}$ \\
\hline & & & Off & \\
\hline & & & Balmy & Balmy \\
\hline & & & Uncommon & Uncommon \\
\hline & & & Outre & \\
\hline & & & Crackbrained & Balanced \\
\hline & & & & Acceptable \\
\hline & & & Balanced & Plain \\
\hline & & & $(5.6 \%)$ & $(5.6 \%)$ \\
\hline$(31 \%)$ & $(38.9 \%)$ & $(45.83 \%)$ & $(51 \%)$ & $(54.3 \%)$ \\
\hline
\end{tabular}

Note. $N=41$ sorters. $K=75$ terms. Roman numbers denote factor order. Numbers in parenthesis indicate the percentage of variance accounted for by that factor and by the entire solution (at the bottom of the table). Arrows indicate whether the factor replicates entirely or splits up in the next factor solution. 
Table 2. Semantic sortings of highly evaluative personality terms (set 2): varimax-rotated solutions and their highest factor markers

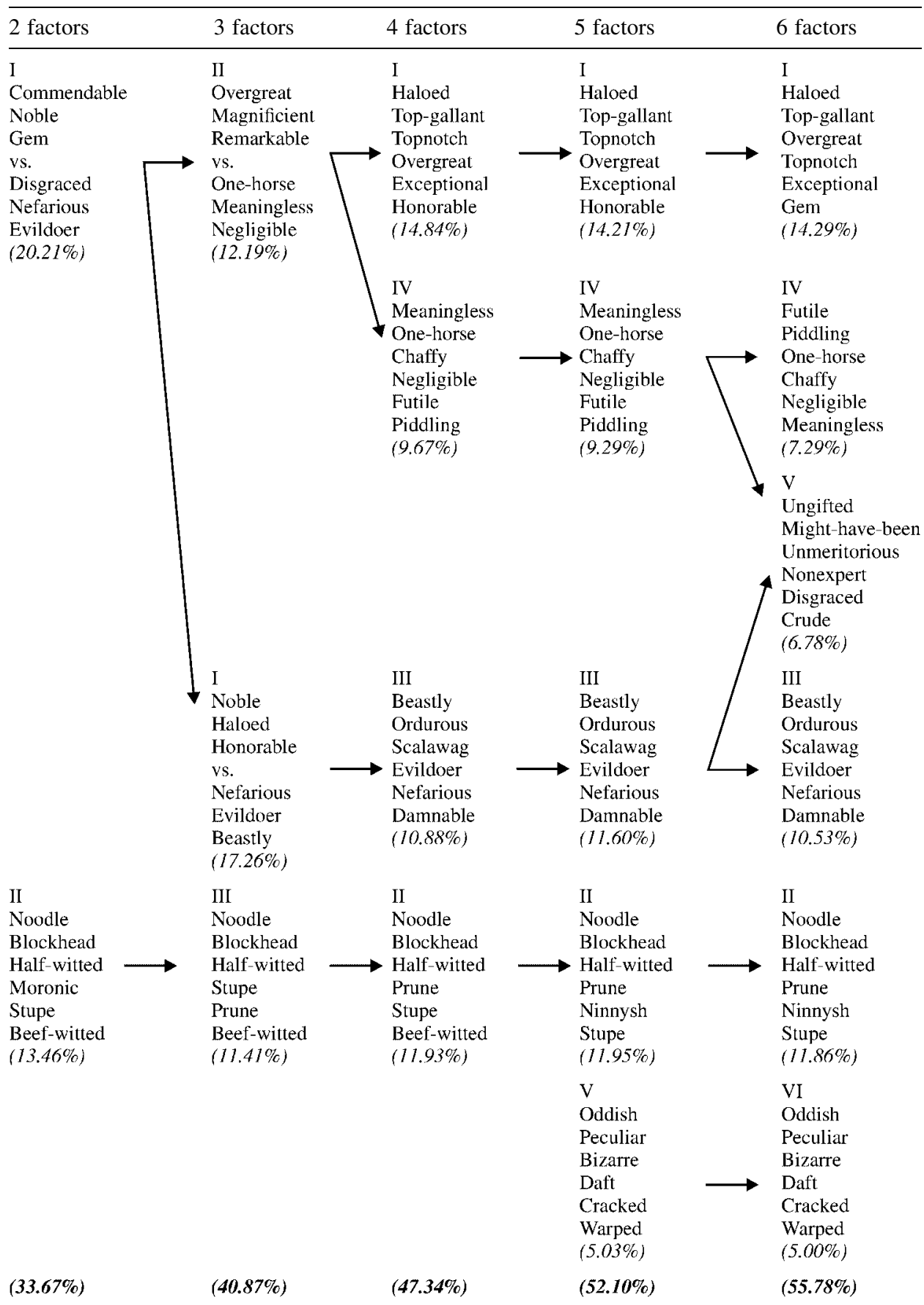

Note. $N=48$ sorters. $K=75$ terms. Roman numbers denote factor order. Numbers in parenthesis indicate the percentage of variance accounted for by that factor or by the entire solution (at the bottom of the table). Arrows indicate whether the factor replicates entirely or splits up in the next factor solution. 
Table 3. Semantic sortings of highly evaluative personality terms (set 3): varimax-rotated solutions and their highest factor markers

\begin{tabular}{|c|c|c|c|c|}
\hline 2 factors & 3 factors & 4 factors & 5 factors & 6 factors \\
\hline \multirow{22}{*}{$\begin{array}{l}\text { II } \\
\text { Paragon } \\
\text { Virtuoso } \\
\text { First-rate } \\
\text { vs. } \\
\text { Brainless } \\
\text { Woodenhead } \\
\text { Doltish } \\
(13.18 \%)\end{array}$} & III & III & III & III \\
\hline & First-rate & First-rate & First-rate & First-rate \\
\hline & Slap-up & Slap-up & Slap-up & Slap-up \\
\hline & Swell & Paragon & Swell & Swell \\
\hline & vs. & vs. & Grand & Grand \\
\hline & Unremarkable & Subnormal & Laureate & Laureate \\
\hline & Indistinctive & Unsatisfactory & Paragon & Paragon \\
\hline & $\begin{array}{l}\text { Middlerate } \\
(10.87 \%)\end{array}$ & $\begin{array}{l}\text { Uncapable } \\
(10.87 \%)\end{array}$ & $(10.05 \%)$ & $(9.44 \%)$ \\
\hline & & & V & $\mathrm{V}$ \\
\hline & & & Unable & Unable \\
\hline & & & Uncapable & Uncapable \\
\hline & & & Bunglesome & Bunglesome \\
\hline & & & Subnormal & Subnormal \\
\hline & & & Inexperienced & Inexperienced \\
\hline & & & $\begin{array}{l}\text { Unsatisfactory } \\
(6.15 \%)\end{array}$ & $\begin{array}{l}\text { Unsatisfactory } \\
(5.93 \%)\end{array}$ \\
\hline & & II & II & II \\
\hline & Woodenhead & Woodenhead & Woodenhead & Woodenhead \\
\hline & Snipe & Snipe & Snipe & Snipe \\
\hline & Doltish & Doltish & Doltish & Doltish \\
\hline & Cockbrained & Stupe & Stupe & Stupe \\
\hline & Stupe & Brainless & Brainless & Brainless \\
\hline & Brainless & Cockbrained & Cockbrained & Cockbrained \\
\hline \multirow{24}{*}{$\begin{array}{l}\text { I } \\
\text { Damnable } \\
\text { Cursed } \\
\text { Lousy } \\
\text { vs. } \\
\text { Respectable } \\
\text { Unstained } \\
\text { Smirchless } \\
(14.7 \%)\end{array}$} & I & I & I & $\mathrm{VI}$ \\
\hline & Damnable & Damnable & Damnable & Unstained \\
\hline & Cursed & Cursed & Cursed & Smirchless \\
\hline & Lousy & Lousy & Lousy & Undepraved \\
\hline & vs. & vs. & & Undebased \\
\hline & Respectable & Respectable & Respectable & Authentic \\
\hline & Smirchless & Smirchless & Smirchless & Unflawed \\
\hline & $\begin{array}{l}\text { Unstained } \\
(14.08 \%)\end{array}$ & $\begin{array}{l}\text { Unstained } \\
(13.13 \%)\end{array}$ & $\begin{array}{l}\text { Unstained } \\
(12.94 \%)\end{array}$ & $(5.02 \%)$ \\
\hline & & & & I \\
\hline & & & & Damnable \\
\hline & & & & Cursed \\
\hline & & & & Lousy \\
\hline & & & & $\begin{array}{l}\text { Derisible } \\
\text { Baseborn }\end{array}$ \\
\hline & & & & Inglorious \\
\hline & & IV & IV & IV \\
\hline & & Distinct & Distinct & Distinct \\
\hline & & Odd & Odd & Odd \\
\hline & & Off & Off & Off \\
\hline & & Anomalous & Anomalous & Anomalous \\
\hline & & vs. & & \\
\hline & & Unremarkable & Unremarkable & Unremarkable \\
\hline & & Indistinct & Indistinct & Indistinct \\
\hline & & Characterless & Characterless & Characterles \\
\hline & & $(7.60 \%)$ & $(7.56 \%)$ & $(7.52 \%)$ \\
\hline$(27.95 \%)$ & $(36.30 \%)$ & $(42.81 \%)$ & $(47.26 \%)$ & $(51.08 \%)$ \\
\hline
\end{tabular}

Note. $N=41$ sorters. $K=75$ terms. Roman numbers denote factor order. Numbers in parenthesis indicate the percentage of variance accounted for by that factor or by the entire solution (at the bottom of the table). Arrows indicate whether the factor replicates entirely or splits up in the next factor solution. 
(but not easily observable in the tables) was that most markers of $(-)$ Conventionality (e.g. oddish, bizarre, distinct) had loadings close to zero in the two- and three-factor solutions, suggesting the need to extract solutions with higher numbers of factors; in fact, these markers did not define a Conventionality dimension until five factors (in sets 1 and 2) and four factors (set 3 ) were extracted. ${ }^{10}$

Perhaps the most important (and visible) fact in the progression of factor solutions depicted in Tables 1 to 3 is the structural and conceptual convergence across sets reached by the five-factor solutions (note that in the two-, three-, four-, and six-factor solutions the overlap across sets is only partial). This convergence is noteworthy given the small to moderate differences in content representation that probably exist among the three sets of terms. This finding pointed to orthogonal structures with five factors as meaningful solutions for the data. These solutions are depicted in Tables 4 to 6 .

Solutions obtained with promax- and quartimax rotation methods did not prove very different from the Varimax-rotated solutions, and the factor inter-correlations tended to be very low. For instance, the mean factor inter-correlations for the oblique five-factor solutions were 0.12 (absolute value) for set $1,0.12$ for set 2, and 0.10 for set 3. Quartimaxrotated solutions failed at producing a first general factor.

All in all, results support the adequacy of the five-factor solutions depicted in Tables 4 to 6 to describe the conceptual relations among our highly evaluative personality adjectives. A striking feature of these solutions is how remarkably similar they are in their factor definitions. Each structure includes factors that purely or partially capture the unique, evaluative elements of the Big Seven factor model (i.e. include evaluative terms related to Positive Valence, Negative Valence, and Conventionality), namely (a) a factor denoting high exceptionality, status, and uniqueness (e.g. pluperfect, haloed, first-rate), which we named distinction; (b) a factor resembling Negative Valence and denoting depravity (e.g. bounder, beastly, damnable), which we labelled depravity; (c) a factor that mainly (but not exclusively) captures the opposite pole of Conventionality (e.g. off, oddish, distinctive), which we called unconventionality; and (d) a factor representing unworthiness (e.g. nonessential, meaningless) in sets 1 and 2, and inability in set 3 (unable), which we labelled worthlessness. ${ }^{11}$

At first glance, the worthlessness dimension may resemble the low pole of distinction, but several features of this dimension weigh against that conclusion: (a) the psychometric independence of this factor from distinction, as suggested by the lack of cross-loadings between the two factors (there are only four cross-loadings equal to or larger than 0.30); and (b) some subtle differences in meaning — note that distinction, as depicted in Tables 4 to 6 , is primarily defined by terms denoting high class and unsurpassability (i.e. social visibility); worthlessness, on the other hand, is represented by terms denoting (low) value (i.e. low social merit or utility). All in all, we are inclined towards an interpretation of distinction and worthlessness as two different facets of Positive Valence.

\footnotetext{
${ }^{10}$ Detailed information regarding all the solutions summarized in Tables 1 to 3 can be obtained from the first author.

${ }^{11}$ We realize that the label worthlessness may not be ideal for the fifth factor in set 3 , which is represented mostly by terms denoting inability (e.g. unable, uncapable), rather than worthlessness (interestingly, note that unconsequential and nonessential, which are markers of worthlessness in set 1, appear as markers of low unconventionality in set 3 ). However, the fact that a clear worthlessness dimension also emerged in study 2 (where all three sets were combined in one measure) suggests that the somewhat different definition of this dimension in Table 3 (see also Table 6) may have been caused by an underepresentation of worthlessness markers in set 3 (relative to sets 1 and 2).
} 
Table 4. Semantic sortings of highly evaluative personality terms (set 1): varimax-rotated five factor solution

\begin{tabular}{|c|c|c|c|c|c|}
\hline Items & Distinction & Depravity & Worthlessness & Stupidity & Unconventionality \\
\hline Pluperfect & 85 & -12 & -05 & -05 & 00 \\
\hline Demigod & 84 & -13 & -03 & -10 & 01 \\
\hline Overgreat & 81 & -15 & -06 & -06 & 00 \\
\hline High-calibre & 81 & -07 & -05 & -19 & -01 \\
\hline First-class & 81 & -03 & -03 & -12 & -02 \\
\hline Faultless & 78 & -16 & -06 & -04 & 00 \\
\hline Unflawed & 76 & -20 & -01 & 04 & -02 \\
\hline Supereminent & 73 & -19 & -06 & -10 & -01 \\
\hline Paralleless & 72 & -06 & -16 & -08 & 05 \\
\hline Excelled & 72 & -02 & -01 & -23 & -04 \\
\hline Sublimish & 72 & -17 & 01 & -13 & 00 \\
\hline Stellar & 71 & -16 & 00 & -22 & 00 \\
\hline Proficient & 62 & -17 & -06 & -29 & -02 \\
\hline Unparalleable & 60 & 01 & -10 & -04 & 09 \\
\hline Stainless & 60 & -26 & 04 & 05 & -02 \\
\hline Respectworthy & 53 & -32 & -18 & -06 & -02 \\
\hline Unmarred & 48 & -24 & 03 & 06 & -03 \\
\hline Unsmirched & 42 & -29 & -07 & 06 & -04 \\
\hline Engraced & 24 & -23 & -13 & -12 & -04 \\
\hline Third-class & -33 & 05 & 08 & 18 & 01 \\
\hline Might-have-been & -33 & -05 & 13 & 21 & 01 \\
\hline Fallible & -42 & -01 & 08 & 41 & 01 \\
\hline Unexemplary & -45 & 10 & 27 & 16 & -02 \\
\hline Unexcellent & $-\mathbf{5 0}$ & 09 & 23 & 14 & -01 \\
\hline Unideal & -51 & 09 & 30 & 11 & 00 \\
\hline Bounder & -12 & 84 & -04 & 04 & 04 \\
\hline Arrant & -11 & 83 & -02 & 02 & 02 \\
\hline Repulsive & -08 & 78 & -02 & 06 & 05 \\
\hline Scoundrel & -27 & 77 & -03 & -03 & 00 \\
\hline Rat & -22 & 75 & -03 & 00 & 00 \\
\hline Buzzard & -09 & 74 & -04 & 04 & 14 \\
\hline Puckish & -08 & 71 & -02 & 01 & 01 \\
\hline Lousy & -12 & 70 & -04 & 10 & 05 \\
\hline Base-minded & -18 & 64 & 04 & 13 & 02 \\
\hline Contemptible & -29 & 63 & 05 & -06 & 02 \\
\hline Fallen & -22 & 54 & 10 & -06 & 03 \\
\hline Disagreeable & 02 & 54 & 01 & 03 & 08 \\
\hline Disgraced & -18 & 54 & 16 & -03 & 00 \\
\hline Scummy & -36 & 52 & 22 & -05 & -01 \\
\hline Honourless & -31 & 51 & 18 & -03 & 00 \\
\hline Denigrated & -28 & 50 & 18 & 01 & 02 \\
\hline Ungenuine & -11 & 35 & 13 & 08 & 04 \\
\hline Overluscious & -09 & -14 & -01 & -08 & 06 \\
\hline Unharmful & -04 & -27 & 16 & 13 & -04 \\
\hline Nifty & 02 & -40 & -09 & -24 & -04 \\
\hline Adorable & -05 & -56 & -03 & -12 & -02 \\
\hline Welldoing & 03 & -57 & 02 & -14 & -05 \\
\hline Nonessential & -12 & 00 & 93 & 00 & 00 \\
\hline Unconsequential & -04 & 04 & 91 & 01 & 03 \\
\hline Inessential & -07 & 03 & 88 & 01 & 01 \\
\hline Pointless & -14 & 03 & 85 & 08 & -01 \\
\hline
\end{tabular}


Table 4. (Continued)

\begin{tabular}{|c|c|c|c|c|c|}
\hline Items & Distinction & Depravity & Worthlessness & Stupidity & Unconventionality \\
\hline Unnecessary & -02 & -03 & 84 & -02 & 00 \\
\hline Cipher & -12 & 09 & 84 & 06 & 04 \\
\hline Null & -15 & -02 & 80 & 07 & -03 \\
\hline Piddling & 01 & 07 & 73 & 00 & 10 \\
\hline Worthless & $-\mathbf{3 0}$ & 21 & 49 & 01 & 02 \\
\hline Plain & -04 & -03 & 36 & 13 & $-\mathbf{3 3}$ \\
\hline Considerable & 22 & -12 & -51 & -10 & -07 \\
\hline Blockheaded & -13 & 02 & 00 & 92 & 03 \\
\hline Blunderhead & -10 & 04 & 00 & 92 & 02 \\
\hline Gooney & -15 & 02 & 02 & 90 & 00 \\
\hline Lunkhead & -17 & 08 & 03 & 90 & 04 \\
\hline Half-witted & -12 & 07 & 03 & 89 & 03 \\
\hline Assish & -09 & 13 & -02 & 81 & 04 \\
\hline Lumpish & -10 & 09 & 05 & 57 & 02 \\
\hline Loutish & -11 & 12 & 05 & 51 & 05 \\
\hline Inexpert & -32 & -02 & 10 & 48 & 04 \\
\hline Uncapable & -36 & -01 & 13 & 44 & 05 \\
\hline Off & -01 & 04 & 00 & 03 & 85 \\
\hline Balmy & -03 & -01 & 03 & 04 & 85 \\
\hline Uncommon & 05 & 00 & 02 & -02 & 85 \\
\hline Outre & 06 & -01 & 01 & 03 & 83 \\
\hline Crackbrained & 04 & 16 & -01 & 08 & 69 \\
\hline Balanced & 07 & -09 & -03 & -06 & -64 \\
\hline Acceptable & 06 & -13 & -07 & -08 & -49 \\
\hline
\end{tabular}

Note. $N=41$ sorters. $K=75$ terms. All loadings multiplied by 100 ; loadings $10.30 \mid$ or larger are set in bold.

Table 5. Semantic sortings of highly evaluative personality terms (set 2): varimax-rotated five factor solution

\begin{tabular}{lccccc}
\hline Items & Distinction & Stupidity & Depravity & Worthlessness & Unconventionality \\
\hline Haloed & $\mathbf{7 9}$ & -10 & -22 & -05 & 00 \\
Topgallant & $\mathbf{7 8}$ & -11 & -09 & -19 & 03 \\
Topnotch & $\mathbf{7 8}$ & -11 & -06 & -19 & 03 \\
Overgreat & $\mathbf{7 8}$ & -15 & -05 & -23 & 02 \\
Exceptional & $\mathbf{7 7}$ & -10 & -12 & -12 & 04 \\
Gem & $\mathbf{7 6}$ & -04 & -23 & -09 & 01 \\
Honourable & $\mathbf{7 6}$ & -11 & -24 & -04 & -01 \\
Magnificent & $\mathbf{7 3}$ & -20 & -08 & -24 & 03 \\
Noble & $\mathbf{7 3}$ & -09 & -28 & -08 & -02 \\
Commendable & $\mathbf{7 2}$ & 02 & -29 & -11 & 01 \\
Remarkable & $\mathbf{7 1}$ & -08 & 00 & -22 & 04 \\
Loveworthy & $\mathbf{6 4}$ & -01 & -20 & -01 & -02 \\
Unflawed & $\mathbf{6 2}$ & -02 & -21 & -17 & -07 \\
Good & $\mathbf{6 1}$ & -07 & -29 & 01 & -09 \\
Virtuous & $\mathbf{5 9}$ & -04 & -23 & 06 & -14 \\
Unmarred & $\mathbf{5 5}$ & -01 & -27 & -11 & -09 \\
Scrumptious & $\mathbf{5 4}$ & -01 & -08 & -17 & 05 \\
Peachy & $\mathbf{5 4}$ & 00 & -25 & 04 & -03 \\
\hline
\end{tabular}


Table 5. (Continued)

\begin{tabular}{|c|c|c|c|c|c|}
\hline Items & Distinction & Stupidity & Depravity & Worthlessness & Unconventionality \\
\hline Unfallen & 52 & -02 & -08 & -20 & -02 \\
\hline Sensational & 47 & 01 & 02 & -08 & 10 \\
\hline Uncrooked & 42 & 01 & -15 & 03 & -11 \\
\hline Novel & 42 & -09 & -05 & -20 & 37 \\
\hline Noodle & -08 & 98 & -01 & 04 & 02 \\
\hline Blockheaded & -09 & 96 & -01 & 02 & 02 \\
\hline Half-Witted & -07 & 95 & -01 & 06 & 00 \\
\hline Prune & -06 & 94 & 00 & 04 & 02 \\
\hline Ninnyish & -06 & 94 & -01 & 06 & 05 \\
\hline Stupe & -03 & 93 & -01 & 02 & 02 \\
\hline Beef-Witted & -03 & 92 & 00 & 03 & 02 \\
\hline Moronic & -12 & 91 & -01 & 06 & 02 \\
\hline Tomfool & -04 & 88 & 05 & 09 & 06 \\
\hline Bunglesome & -05 & 49 & 03 & 36 & -03 \\
\hline Lacklustre & -26 & 46 & -01 & 30 & -12 \\
\hline Beastly & -12 & 01 & 73 & -06 & 04 \\
\hline Ordurous & -11 & 01 & 71 & 06 & 05 \\
\hline Scalawag & -19 & 04 & 71 & -06 & 03 \\
\hline Damnable & -19 & -01 & 69 & -01 & -04 \\
\hline Evildoer & -27 & -01 & 68 & -08 & 04 \\
\hline Nefarious & -28 & -01 & 66 & -11 & 04 \\
\hline Ignominious & -23 & 10 & 65 & 08 & -02 \\
\hline Disgraceful & -19 & -04 & 63 & 26 & -03 \\
\hline Buzzard & -01 & 04 & 62 & -05 & 07 \\
\hline Wrong-Hearted & -26 & -02 & 61 & 00 & 15 \\
\hline Flagrant & -06 & -03 & 59 & -04 & 12 \\
\hline $\mathrm{Cad}$ & -14 & 01 & 58 & 13 & 03 \\
\hline Deplorable & -07 & -03 & 58 & 09 & 03 \\
\hline Dastardly & -27 & 03 & 58 & 10 & 01 \\
\hline Unadmirable & -20 & -05 & 57 & 25 & -01 \\
\hline Demeaned & -24 & -01 & 53 & 32 & -03 \\
\hline Blot & -11 & -03 & 50 & 26 & 01 \\
\hline Crummy & -07 & 00 & 50 & 31 & 04 \\
\hline Unmeritorious & -18 & 07 & 41 & 33 & 01 \\
\hline Unenviable & -21 & -02 & 40 & 33 & 00 \\
\hline Crude & 01 & 10 & 39 & 27 & -08 \\
\hline Meaningless & -12 & -03 & 09 & 85 & -01 \\
\hline One-Horse & -16 & 02 & 00 & 85 & 01 \\
\hline Chaffy & -08 & 07 & -01 & 79 & -02 \\
\hline Negligible & -13 & 04 & -03 & 78 & 00 \\
\hline Futile & -09 & -01 & -04 & 75 & 08 \\
\hline Piddling & -06 & 04 & -03 & 74 & 09 \\
\hline Good-For-Nothing & -17 & 10 & 14 & 65 & -04 \\
\hline Scummy & -12 & -02 & 33 & 52 & -01 \\
\hline Ungifted & -16 & 25 & 09 & 49 & -03 \\
\hline Might-Have-Been & -24 & 10 & 17 & 47 & 00 \\
\hline Uncapable & -13 & 40 & 03 & 42 & -01 \\
\hline Dislustered & -02 & 05 & 26 & 40 & -11 \\
\hline Nonexpert & -19 & 33 & 02 & 37 & -03 \\
\hline Menial & -06 & 23 & 16 & 36 & -02 \\
\hline Oddish & 01 & -02 & -01 & 01 & 88 \\
\hline Peculiar & 01 & -04 & -01 & 00 & 88 \\
\hline
\end{tabular}


Table 5. (Continued)

\begin{tabular}{lccccc}
\hline Items & Distinction & Stupidity & Depravity & Worthlessness & Unconventionality \\
\hline Bizarre & 01 & -04 & -01 & 04 & $\mathbf{8 6}$ \\
Daft & -04 & 13 & 07 & 00 & $\mathbf{6 9}$ \\
Cracked & -03 & 06 & 11 & 05 & $\mathbf{6 4}$ \\
Warped & -21 & -01 & $\mathbf{4 0}$ & 00 & $\mathbf{4 7}$ \\
Cataclysmal & 01 & -01 & 20 & -08 & 21 \\
\hline
\end{tabular}

Note. $N=49$ sorters. $K=75$ terms. All loadings multiplied by 100 ; loadings 10.301 or larger are set in bold.

Table 6. Semantic sortings of highly evaluative personality terms (set 3): varimax-rotated five factor solution

\begin{tabular}{lrrrrr}
\hline Items & Depravity & Stupidity & Distinction & Unconventionality & Worthlessness \\
\hline Damnable & $\mathbf{8 8}$ & -01 & -12 & -01 & 03 \\
Cursed & $\mathbf{8 8}$ & -01 & -12 & -01 & 03 \\
Lousy & $\mathbf{8 1}$ & -01 & -12 & -01 & 04 \\
Baseborn & $\mathbf{7 8}$ & 00 & -05 & 00 & 05 \\
Derisible & $\mathbf{7 8}$ & 04 & -14 & 00 & 07 \\
Inglorious & $\mathbf{7 6}$ & -02 & -15 & 02 & 06 \\
Dreggy & $\mathbf{7 5}$ & -01 & -03 & -01 & 02 \\
Stained & $\mathbf{7 2}$ & -01 & -17 & -03 & 04 \\
Cattle & $\mathbf{7 0}$ & 09 & -04 & 00 & 02 \\
Insufferable & $\mathbf{7 0}$ & 00 & -09 & 00 & 01 \\
Reprehensible & $\mathbf{6 7}$ & -02 & -18 & 00 & 04 \\
Base & $\mathbf{6 7}$ & -01 & -07 & 01 & 08 \\
Unsufferable & $\mathbf{6 7}$ & 00 & -10 & 00 & 04 \\
Disparageable & $\mathbf{6 3}$ & -03 & -20 & -03 & 14 \\
Flagrant & $\mathbf{6 2}$ & 02 & -04 & 00 & 02 \\
Pill & $\mathbf{4 9}$ & 07 & 02 & 03 & 02 \\
Unbonny & $\mathbf{4 5}$ & -01 & -03 & 00 & 06 \\
Undeserving & $\mathbf{4 2}$ & -02 & -02 & 13 & $\mathbf{3 4}$ \\
Misbeseeming & $\mathbf{3 1}$ & 01 & -05 & 03 & 11 \\
Consequential & 23 & 00 & 09 & -11 & -11 \\
Peachy & -17 & -01 & 15 & 00 & 11 \\
Honey & -28 & -01 & 15 & -01 & 09 \\
Wooden-Headed & 01 & $\mathbf{9 5}$ & -05 & 02 & 12 \\
Snipe & 01 & $\mathbf{9 4}$ & -06 & -03 & 10 \\
Cockbrained & 00 & $\mathbf{9 3}$ & -05 & -02 & 08 \\
Stupe & 01 & $\mathbf{9 3}$ & -05 & 01 & 13 \\
Doltish & 01 & $\mathbf{9 3}$ & -04 & 02 & 14 \\
Brainless & 00 & $\mathbf{9 2}$ & -07 & 06 & 15 \\
Blear-Witted & 00 & $\mathbf{8 9}$ & -06 & 08 & 12 \\
Goosish & 01 & $\mathbf{8 8}$ & -06 & -02 & 07 \\
Gump & 00 & $\mathbf{8 7}$ & -06 & 11 & -14 \\
First-Rate & -04 & -04 & $\mathbf{8 5}$ & -06 & -17 \\
Slap-Up & -06 & -04 & $\mathbf{7 9}$ & -05 & -13 \\
Swell & -08 & -02 & $\mathbf{7 7}$ & -09 & -04 \\
Grand & -02 & 00 & $\mathbf{7 5}$ & -08 & -05 \\
Laureate & -06 & -05 & $\mathbf{7 3}$ & -01 & -25 \\
Paragon & 01 & -09 & $\mathbf{7 3}$ & -02 & $C o n t h y s$ \\
\hline & & & & & \\
\hline
\end{tabular}


Table 6. (Continued)

\begin{tabular}{|c|c|c|c|c|c|}
\hline Items & Depravity & Stupidity & Distinction & Unconventionality & Worthlessness \\
\hline Respectable & -23 & 01 & 64 & 02 & -02 \\
\hline Unflawed & -10 & -02 & 60 & 01 & -12 \\
\hline Demigod & 02 & -10 & 57 & -05 & -24 \\
\hline Unparagoned & -03 & -06 & 56 & -24 & -13 \\
\hline Virtuoso & 00 & -10 & 54 & -04 & -36 \\
\hline Extraordinary & -03 & -10 & 50 & -43 & -03 \\
\hline Undebased & -22 & -03 & 46 & 09 & 15 \\
\hline Unstained & -28 & 02 & 46 & 00 & 10 \\
\hline Smirchless & -29 & 02 & 44 & -01 & 07 \\
\hline Prowessfull & -16 & 01 & 41 & -06 & -08 \\
\hline Undepraved & -32 & 00 & 40 & 06 & 18 \\
\hline Abundant & 00 & -03 & 40 & -02 & -21 \\
\hline Swellish & -11 & -05 & 37 & -07 & -09 \\
\hline Competent & -03 & -09 & 37 & 01 & -32 \\
\hline Authentic & -23 & 00 & 26 & 02 & 08 \\
\hline Pithsome & -01 & 02 & 15 & -03 & -11 \\
\hline Unremarkable & 00 & 00 & -06 & 83 & 04 \\
\hline Indistinctive & 00 & 02 & -05 & 82 & 02 \\
\hline Characterless & 04 & 02 & -04 & 73 & 08 \\
\hline Middle-Rate & 00 & -03 & -05 & 71 & 13 \\
\hline Unmomentous & 06 & -01 & 02 & 69 & 18 \\
\hline Unconsequential & 02 & -01 & 03 & 67 & 20 \\
\hline Nonessential & 01 & 00 & 02 & 66 & 21 \\
\hline Barren & 04 & 32 & 00 & 52 & 09 \\
\hline Unmarvellous & 12 & -04 & -20 & 41 & 35 \\
\hline Anomalous & 21 & -03 & -01 & -32 & 20 \\
\hline Off & 05 & -02 & 07 & -48 & 24 \\
\hline Odd & 04 & -01 & 07 & -52 & 20 \\
\hline Distinctive & 05 & -02 & 21 & -70 & 08 \\
\hline Unable & -01 & 12 & -07 & 04 & 74 \\
\hline Uncapable & -01 & 11 & -08 & 01 & 72 \\
\hline Bunglesome & -04 & 20 & -13 & 01 & 68 \\
\hline Inexepert & -04 & 21 & -12 & 04 & 61 \\
\hline Subnormal & 13 & 07 & -17 & -02 & 61 \\
\hline Unsatisfactory & 09 & -01 & -17 & 12 & 59 \\
\hline Lackall & 06 & 14 & -10 & 15 & 58 \\
\hline Faultful & 14 & 03 & -15 & -01 & 50 \\
\hline Rotter & 21 & 22 & -05 & 01 & 28 \\
\hline
\end{tabular}

Note. $N=41$ sorters. $K=75$ terms. All loadings multiplied by 100 ; loadings $10.30 \mid$ or larger are set in bold. ${ }^{\mathrm{R}}$ Reversed.

The five-factor solutions depicted in Tables 4 to 6 also include a factor denoting low intelligence (e.g., blockheaded, noodle, woodenheaded), which we named Stupidity. Not surprisingly, this factor has never emerged in previous studies of the Big Seven (Almagor et al., 1995; Benet-Martínez, 1999; Tellegen and Waller, 1987) due to the exclusion of cognitive-ability descriptors in this and other lexical work (e.g. Angleitner, Ostendorf and John, 1990).

Other noticeable features in the five-factor structures are the largely mono-polar nature of most dimensions (with the exceptions of distinction and depravity in set 1 , and unconventionality in sets 1 and 3), the large size of the distinction and depravity dimensions, and the high degree of simple structure in the factor loadings. Differences 
among the three structures in factor order suggest small discrepancies in content saturation among the three sets. This is not surprising given that the 75 terms from each set were randomly selected from Norman's original pool of 760 adjectives, a procedure that unavoidably may have led to an unequal sampling of person descriptive domains in each set. This difference, nevertheless, does not override the substantial factorial overlap found across the three sets. In fact, a close inspection of the across-sets consistency of the primary loadings for those 21 terms that were repeated in more than one set confirms this conclusion: only seven of these repeated items had primary loadings that were not perfectly consistent across sets, (bunglesome, inexpert, might-have-been, unconsequential, nonessential, scummy, and uncapable), although three of these terms (nonessential, scummy, and uncapable) had secondary loadings on the expected dimensions.

In summary, results from study 1 suggest that dimensions representing distinction, worthlessness, depravity, stupidity, and unconventionality describe the conceptual (i.e. semantic) relations among our randomly selected highly evaluative personality descriptors.

\section{STUDY 2: EXTERNAL STRUCTURE OF HIGHLY EVALUATIVE PERSONALITY DESCRIPTORS}

The goal of study 1 was to explore people's implicit theories about the conceptual relations among highly evaluative personality adjectives (i.e. internal structure). In study 2 we explored the actual covariation in people of the dispositions represented by these terms (i.e. external structure).

\section{Method}

\section{Sample}

A combined sample of 180 undergraduate students (62 men and 118 women) with a mean age of 20.75 years $(S D=1.9)$ from the University of California at Berkeley $(\mathrm{N}=50)$ and the University of Michigan (130) participated in our second study. English was the first language of all participants. A wide range of majors was represented but the majority of the participants were psychology majors.

\section{Measures and procedure}

Recall that in study 1 we used three sets of 75 adjectives so that the sorting exercise would not be excessively tiring to our participants. As suggested by the results from the sorting study, one limitation of this procedure (using three different pools of terms) is the unequal sampling of content domains across the three sets. To prevent this problem in the selfreports, terms from the three sets $(K=225)$ were combined into a single measure (with three sections corresponding to each set) and presented in alphabetical order. As in study 1 , each term was followed by a synonym or short definition (identical to that used in study 1), and subjects provided self-ratings using a seven-point Likert scale.

Participants were tested in small groups during class (University of Michigan) or experimental sessions (University of California at Berkeley). They were given the following instructions:

Your next task is to consider a number of descriptors as they may or may not apply to you. Please, read each statement carefully and respond to each with the appropriate 
number. Rate each attribute as it applies to the way you are generally or typically, as compared with other persons you know of the same sex and age. Fill in only one response for each statement.

Self-ratings provided by participants were based on a scale that ranged from 1 (strongly disagree) to 7 (strongly agree).

\section{Results and discussion}

Elucidating the underlying structure of self-reports on our pool of highly evaluative adjectives presents a number of methodological challenges that did not apply to the semantic sortings conducted in study 1 . First, due to the high (un-) desirability of many of the adjectives, we suspected that several of the young college students may have provided less than accurate self-portrayals. Thus, care was taken to screen the data for obvious random response patterns and invalid protocols. This was accomplished by creating a 23 -item response inconsistency scale (Baer, Kroll, Rinaldo and Ballenger, 1999; Tellegen, unpublished manuscript) from the 21 words that were repeated more than once in the three sets from study 1 (recall than two of these 21 words appeared three times). More formally, the response inconsistency scale was created by summing the absolute differences in the item responses to the 23 pairs of repeated words. The observed distribution of this aggregate was right skewed - as expected because we summed absolute difference scores. Several scores were noticeably higher than the rest. The scores on our validity scale ranged from 0 to 59. The median score was eight and the median absolute deviation score (a robust estimate of the scale, which is similar in spirit to the standard deviation, but more informative for highly skewed response distributions) was 4.45. Response protocols were deemed invalid if a person's response inconsistency score was greater than or equal to 15 (this value represents the median plus 1.5 times the mean absolute deviation). Eleven of the 180 persons with complete data were excluded by this purposely conservative exclusion criterion.

\section{Factor analyses}

To avoid the inclusion of repeated variables in the factor analyses (remember that 19 terms appeared in two sets and two terms appeared in all three sets), scores representing the mean response across sets for each repeated term were used. This procedure left us with a final matrix of 202 variables (from the initial set of 225). Prior to the factor analyses, responses were ipsatized (i.e. within-subject standardized) to control for the potential influences of individual differences in subjects' (un-) willingness to endorse the extreme values of the response categories (Hamilton, 1968). In our experience (Benet and Waller, 1995; Benet-Martínez and Waller, 1997) and the experience of other researchers in this area (Goldberg, 1992; Hamilton, 1968; Peabody and Goldberg, 1989; Yang and Bond, 1990), ipsatizing scores prior to multivariate analyses frequently yields cleaner (better defined simple structure) factor patterns.

The first ten eigenvalues for the $202 \times 202$ correlations were $30,14,11.7,10.6,9,7,6$, 5.6, 5.5, and 5. As in study 1, we examined varimax-, promax-, and quartimax-rotated solutions with two to six factors. ${ }^{12}$ Selection of the final dimensional structures was guided by the scree plot, the psychological coherence of the obtained solutions, and the residual plots and goodness-of-fit indices for different solutions. We discuss the varimax-rotated solutions first.

\footnotetext{
${ }^{12}$ Note that the $N / k$ ratio of this study $(169 / 202=0.84)$ is quite small. Given the high degree of content saturation of our variables (i.e. the great redundancy among the 202 descriptors), this should not be a big problem (see Guadagnoli and Verlicer, 1988; for a discussion and illustration of the importance of component saturation and absolute sample size, over $N / k$ ratio, in predicting factor stability).
} 
The two-factor solution included a very large positive valence dimension plus a conventionality factor. The three-factor solution included dimensions representing positive valence, stupidity, and conventionality (note: in the two- and three-factor solutions most depravity terms had loadings smaller than 0.35 ). The four-factor solution included distinction, worthlessness, unconventionality, and a dimension mixing depravity and stupidity. The five-factor solution included dimensions that were conceptually narrower and represented distinction, stupidity, worthlessness, unconventionality, and depravity. The six-factor solution included two worthlessness factors (which shared many cross-loadings). The seven-factor solution was like the six-factor solution plus a small factor representing trashiness (e.g. crummy, ordurous).

The promax- and quartimax-rotated solutions were very similar to the varimax-rotated solutions. The oblique five-factor solution, for instance, had factor inter-correlations that were very low: the mean factor-intercorrelation was 0.11 (absolute value), and the highest correlation was -0.30 between distinction and stupidity. These results, in consideration with the psychological coherence of the previously described orthogonal solutions, the scree plot, and the residual plots and goodness-of-fit indices for different solutions pointed to the varimax-rotated five-factor solution as appropriate for the data. This solution, which accounted for $34 \%$ of the total variance, is reported in Table 7.

Table 7. Self-reports of highly-evaluative personality terms: varimax-rotated five factor solution

\begin{tabular}{|c|c|c|c|c|c|}
\hline Items & Distinction & Stupidity & Worthlessness & Unconventionality & Depravity \\
\hline Top-gallant & 81 & -13 & 10 & -01 & 06 \\
\hline Topnotch & 78 & -11 & 07 & 02 & 10 \\
\hline Overgreat & 73 & -17 & 05 & 06 & -04 \\
\hline Sublimish & 71 & -14 & -12 & 10 & -09 \\
\hline Paragon & 68 & -15 & -12 & 09 & 03 \\
\hline Grand & 67 & -24 & -06 & 10 & 00 \\
\hline Pluperfect & 65 & -12 & -03 & -04 & -18 \\
\hline Remarkable & 64 & -06 & -04 & 10 & 06 \\
\hline Unflawed & 62 & -07 & -02 & 21 & -35 \\
\hline Stellar & 62 & -25 & -01 & 07 & 08 \\
\hline Demigod & 61 & -04 & -06 & -12 & -05 \\
\hline First-rate & 61 & -22 & -14 & 21 & -04 \\
\hline Magnificent & 59 & -22 & -20 & 08 & 03 \\
\hline Slap-up & 58 & -17 & -26 & 20 & -11 \\
\hline Overluscious & 57 & -12 & -14 & 01 & 17 \\
\hline Swell & 54 & -24 & -07 & 24 & -13 \\
\hline Laureate & 51 & -05 & -16 & 14 & 07 \\
\hline Parallelless & 51 & -15 & 08 & -21 & 01 \\
\hline Supereminent & 51 & -12 & -22 & 14 & 02 \\
\hline Haloed & 49 & -06 & 01 & 07 & -01 \\
\hline Virtuoso & 49 & -04 & -11 & 15 & -22 \\
\hline Faultless & 49 & -12 & -12 & 18 & -11 \\
\hline First-class & 47 & -40 & -05 & 17 & 12 \\
\hline Scrumptious & 47 & -18 & -33 & 07 & -17 \\
\hline Unparagoned & 45 & -21 & -03 & 33 & 02 \\
\hline Extraordinary & 45 & -21 & -20 & 11 & -01 \\
\hline High-calibre & 44 & -26 & -01 & 26 & 06 \\
\hline Smirchless & 44 & -15 & -06 & -30 & -36 \\
\hline Prowessful & 43 & 00 & -17 & -13 & 07 \\
\hline
\end{tabular}


Table 7. (Continued)

\begin{tabular}{|c|c|c|c|c|c|}
\hline Items & Distinction & Stupidity & Worthlessness & Unconventionality & Depravity \\
\hline Exceptional & 42 & -12 & -07 & 29 & 19 \\
\hline Proficient & 40 & -21 & 10 & 05 & 01 \\
\hline Unparallellable & 38 & -21 & 05 & 30 & 05 \\
\hline Excelled & 35 & -32 & 06 & 14 & 19 \\
\hline Stainless & 34 & 04 & -10 & -27 & -33 \\
\hline Virtuous & 33 & 12 & -22 & -28 & -15 \\
\hline Honorable & 28 & -15 & -05 & 03 & -10 \\
\hline Authentic & 26 & 13 & -23 & 26 & -12 \\
\hline Sensational & 25 & 09 & -21 & -01 & -16 \\
\hline Plain & -27 & 21 & 20 & -24 & -09 \\
\hline Unable & -34 & 31 & 26 & -22 & 04 \\
\hline Uncapable & -35 & 11 & 32 & 04 & -08 \\
\hline Piddling & -36 & -18 & 04 & 06 & -02 \\
\hline Might-have-been & -37 & -05 & 24 & -07 & -04 \\
\hline Lumpish & -38 & -10 & 14 & -24 & -20 \\
\hline Unexcellent & -39 & 31 & 07 & -05 & -09 \\
\hline Faultful & -40 & 07 & 11 & -10 & 24 \\
\hline Bunglesome & -41 & 18 & 15 & 00 & -13 \\
\hline Acceptable & -43 & -08 & -31 & -40 & -06 \\
\hline Unsatisfactory & -44 & 07 & 31 & -07 & 07 \\
\hline Loutish & -44 & -13 & 03 & -28 & -15 \\
\hline Unexemplary & -49 & 22 & 16 & 04 & 37 \\
\hline Fallible & -50 & 02 & 03 & -11 & -01 \\
\hline Middle-rate & -51 & -07 & 28 & 01 & -15 \\
\hline Inexepert & -52 & -12 & 39 & 13 & -06 \\
\hline Unremarkable & -52 & -19 & 36 & 18 & -13 \\
\hline Unmarvelous & -52 & 22 & 02 & -01 & -03 \\
\hline Undeserving & -55 & 03 & 25 & 17 & -03 \\
\hline Unenviable & -58 & 00 & 37 & 19 & 04 \\
\hline Nonexpert & -60 & -03 & 02 & 06 & -03 \\
\hline Unideal & -64 & 04 & 01 & -08 & -16 \\
\hline Wooden-headed & -19 & 82 & 05 & -04 & -06 \\
\hline Lunkhead & -18 & 81 & 05 & -04 & 02 \\
\hline Half-witted & -17 & 77 & -08 & -04 & -20 \\
\hline Blockheaded & -20 & 75 & 08 & -01 & -19 \\
\hline Beef-witted & -19 & 74 & 09 & 17 & -06 \\
\hline Snipe & -08 & 73 & -08 & -13 & -08 \\
\hline Noodle & -24 & 71 & 21 & -04 & -04 \\
\hline Stupe & -06 & 71 & 04 & 07 & -15 \\
\hline Prune & -15 & 66 & 02 & -18 & -03 \\
\hline Moronic & -19 & 64 & 01 & -04 & -25 \\
\hline Brainless & -25 & 59 & -04 & 25 & 14 \\
\hline Cipher & -19 & 57 & 26 & -03 & -24 \\
\hline Gump & -09 & 56 & 26 & 05 & 15 \\
\hline Ninnyish & -19 & 56 & 16 & -03 & 12 \\
\hline Doltish & -16 & 54 & -02 & -05 & 04 \\
\hline Blear-witted & -24 & 54 & 05 & 22 & 14 \\
\hline Good-for-nothing & -19 & 46 & 27 & -04 & -05 \\
\hline Gooney & -09 & 43 & -02 & -25 & -25 \\
\hline Blunderhead & -10 & 42 & -09 & 16 & -16 \\
\hline Lousy & -24 & 40 & 23 & -04 & 09 \\
\hline Cockbrained & -27 & 39 & -03 & 31 & -10 \\
\hline
\end{tabular}


Table 7. (Continued)

\begin{tabular}{|c|c|c|c|c|c|}
\hline Items & Distinction & Stupidity & Worthlessness & Unconventionality & Depravity \\
\hline Scummy & -03 & 37 & 19 & 27 & 15 \\
\hline Lackall & -17 & 32 & 24 & -02 & -04 \\
\hline Rotter & -25 & 27 & -04 & -03 & 19 \\
\hline Crude & -19 & 22 & 12 & -18 & -02 \\
\hline Third-class & -15 & 16 & 09 & 10 & -11 \\
\hline Pill & -09 & -19 & -05 & 14 & 10 \\
\hline Consequential & 16 & -22 & -17 & -21 & 19 \\
\hline Honourless & -06 & -22 & 10 & 05 & -01 \\
\hline $\mathrm{Cad}$ & -13 & -22 & 22 & 09 & 03 \\
\hline Base-minded & -20 & -24 & -19 & 00 & 18 \\
\hline Buzzard & -03 & -27 & -20 & 16 & 13 \\
\hline Disagreeable & -11 & -36 & -10 & -08 & 14 \\
\hline Unconsequential & -30 & 09 & 74 & 17 & 08 \\
\hline Unmomentous & -32 & 04 & 74 & 12 & -08 \\
\hline Inessential & -15 & 11 & 67 & -07 & -10 \\
\hline Meaningless & -10 & 16 & 61 & 11 & 02 \\
\hline One-horse & -40 & 09 & 58 & 13 & -02 \\
\hline Nonessential & -47 & -06 & 58 & -09 & -06 \\
\hline Unnecessary & -20 & -05 & 55 & 00 & -40 \\
\hline Lackluster & -43 & -09 & 53 & 17 & -04 \\
\hline Subnormal & -17 & 29 & 53 & 12 & 37 \\
\hline Barren & -35 & 11 & 52 & 25 & -03 \\
\hline Unmeritorious & -38 & 04 & 47 & 12 & 16 \\
\hline Ungifted & -42 & -07 & 46 & 04 & -16 \\
\hline Dislustred & -30 & -08 & 43 & 00 & 04 \\
\hline Pointless & -21 & 13 & 41 & -37 & -33 \\
\hline Negligible & -24 & -05 & 39 & -35 & 14 \\
\hline Unbonny & -30 & 29 & 38 & 07 & 03 \\
\hline Chaffy & -34 & 37 & 37 & 01 & -26 \\
\hline Ungenuine & -08 & 04 & 37 & 10 & 12 \\
\hline Unadmirable & -21 & 04 & 33 & 08 & 15 \\
\hline Insufferable & 02 & 21 & 29 & -12 & 18 \\
\hline Menial & 07 & -02 & 29 & 25 & 20 \\
\hline Crummy & 00 & -02 & 24 & 12 & 23 \\
\hline Repulsive & 02 & 11 & 20 & -12 & 10 \\
\hline Scalawag & -10 & -02 & -16 & 06 & 13 \\
\hline Assish & 14 & 12 & -20 & 07 & -18 \\
\hline Cattle & 06 & 21 & -22 & 00 & 14 \\
\hline Derisible & 06 & 00 & -23 & -02 & 11 \\
\hline Baseborn & 00 & 01 & -26 & 07 & 24 \\
\hline Contemptible & -07 & 17 & -27 & -08 & 07 \\
\hline Pithsome & -03 & -27 & -29 & -15 & 18 \\
\hline Abundant & -15 & -05 & -29 & 12 & 08 \\
\hline Respectworthy & 34 & 06 & -35 & 29 & 09 \\
\hline Engraced & 05 & -16 & -37 & 26 & 03 \\
\hline Disgraced & -18 & -07 & -38 & 23 & 08 \\
\hline Gem & 40 & -09 & -40 & 37 & 07 \\
\hline Nifty & 19 & -09 & -47 & 37 & 07 \\
\hline Honey & 04 & -10 & -48 & 30 & -18 \\
\hline Good & 22 & -22 & -52 & 14 & -02 \\
\hline Considerable & 08 & -12 & -61 & 30 & 19 \\
\hline Loveworthy & 31 & -14 & -62 & 14 & 05 \\
\hline
\end{tabular}


Table 7. (Continued)

\begin{tabular}{|c|c|c|c|c|c|}
\hline Items & Distinction & Stupidity & Worthlessness & Unconventionality & Depravity \\
\hline Swellish & 33 & 00 & -64 & 22 & -08 \\
\hline Odd & 23 & -09 & -01 & 77 & 00 \\
\hline Oddish & 12 & -08 & 12 & 75 & 13 \\
\hline Bizarre & 11 & -11 & 09 & 72 & -02 \\
\hline Balmy & 00 & -09 & 01 & 70 & -13 \\
\hline Outre & 04 & 00 & -11 & 68 & -06 \\
\hline Daft & 17 & 08 & -02 & 67 & 03 \\
\hline Off & 15 & -14 & 09 & 65 & 15 \\
\hline Peculiar & 20 & -11 & 10 & 64 & 10 \\
\hline Anomalous & 11 & -01 & 14 & 60 & 23 \\
\hline Cataclysmal & -07 & 03 & -08 & 57 & -04 \\
\hline Uncommon & 15 & 03 & -03 & 55 & 14 \\
\hline Crackbrained & 13 & 09 & -06 & 44 & 34 \\
\hline Cracked & 29 & 08 & -09 & 42 & -05 \\
\hline Goosish & 18 & 15 & -18 & 38 & -13 \\
\hline Tomfool & 16 & 23 & -17 & 37 & 02 \\
\hline Novel & 22 & -21 & -18 & 35 & 08 \\
\hline Misbeseeing & 00 & 03 & -02 & 26 & 11 \\
\hline Distinctive & 04 & -07 & -21 & 24 & 04 \\
\hline Denigrated & 11 & 11 & -04 & -11 & 00 \\
\hline Adorable & 17 & 18 & -25 & -35 & -14 \\
\hline Balanced & -09 & -22 & -14 & -37 & -01 \\
\hline Competent & 12 & -26 & -34 & -41 & 11 \\
\hline Respectable & 10 & 11 & -37 & -46 & 01 \\
\hline Well-doing & 43 & 14 & -28 & -48 & -24 \\
\hline Indistinctive & -25 & -04 & 21 & -54 & 04 \\
\hline Commendable & -13 & -06 & -32 & -55 & 06 \\
\hline Characterless & 18 & -14 & 10 & -57 & -14 \\
\hline Evildoer & -13 & -11 & -24 & 09 & 62 \\
\hline Damnable & -06 & 13 & 01 & 02 & 61 \\
\hline Rat & -17 & -08 & -17 & 12 & 55 \\
\hline Scoundrel & -18 & -13 & -12 & 05 & 54 \\
\hline Pukish & -27 & -05 & -10 & -04 & 52 \\
\hline Cursed & 01 & -07 & -01 & 11 & 51 \\
\hline Dreggy & 00 & 06 & 33 & -09 & 51 \\
\hline Inglorious & 05 & -10 & -03 & -28 & 48 \\
\hline Demeaned & 19 & 01 & 09 & -17 & 48 \\
\hline Unsufferable & -09 & -01 & 07 & 17 & 47 \\
\hline Nefarious & -14 & -17 & -33 & -05 & 45 \\
\hline Flagrant & -05 & -11 & 00 & 03 & 45 \\
\hline Base & -10 & -21 & -07 & 01 & 45 \\
\hline Wrong-hearted & 06 & -22 & -04 & 11 & 44 \\
\hline Beastly & -08 & 24 & -12 & 09 & 43 \\
\hline Arrant & -23 & -17 & -09 & 00 & 39 \\
\hline Stained & 07 & -10 & -02 & -17 & 37 \\
\hline Ignominious & 09 & 02 & -05 & -13 & 37 \\
\hline Fallen & 17 & -20 & 19 & 01 & 36 \\
\hline Deprolable & -07 & 30 & -12 & -06 & 34 \\
\hline Dastardly & 12 & 14 & -18 & 04 & 34 \\
\hline Warped & 04 & -29 & 17 & 32 & 34 \\
\hline Reprehensible & -28 & -04 & -12 & 05 & 33 \\
\hline Blot & 16 & 14 & -05 & 00 & 31 \\
\hline
\end{tabular}


Table 7. (Continued)

\begin{tabular}{lrrccc}
\hline Items & Distinction & Stupidity & Worthlessness & Unconventionality & Depravity \\
\hline Ordurous & -12 & -07 & -25 & -09 & 31 \\
Disparageable & 13 & 01 & 10 & -21 & 30 \\
Bounder & -07 & -12 & -20 & 02 & 21 \\
Disgraceful & 01 & -03 & 05 & 04 & 08 \\
Noble & 06 & -07 & -13 & -14 & -16 \\
Uncrooked & -05 & 02 & -03 & -06 & -20 \\
Unsmirched & -13 & 04 & 02 & -04 & -19 \\
Unfallen & 01 & -22 & -05 & -05 & -23 \\
Futile & 27 & 14 & 04 & 17 & -28 \\
Worthless & 14 & 25 & 28 & -10 & -31 \\
Null & -28 & 17 & 20 & 08 & -31 \\
Undebased & 15 & 00 & -13 & 04 & -34 \\
Peachy & 14 & 27 & -33 & -21 & -36 \\
Unharmful & 06 & -09 & -22 & -19 & -38 \\
Unmarred & -14 & -20 & -07 & 13 & $-\mathbf{4 0}$ \\
Unstained & 27 & -05 & -08 & -22 & $-\mathbf{4 4}$ \\
Undepraved & 29 & 00 & -10 & -20 & $-\mathbf{4 9}$ \\
\hline
\end{tabular}

Note. $N=169 . K=202$ terms. All loadings multiplied by 100 ; loadings $10.40 \mid$ or larger are set in bold.

A first glance at the five-factor solution reveals the presence of the same five recognizable dimensions that appeared in the three structures reported in study 1 , namely distinction (topgallant, topnotch, versus unideal, nonexpert), stupidity (wooden-headed, lunkhead), worthlessness (unconsequential, unmomentous, versus loveworthy, considerable), unconventionality (characterless, indistinctive, versus odd, bizarre), and depravity (evildoer, damnable, versus undepraved, unstained). Note, however, that unlike the case in the semantic-sorting structures reported for sets 2 and 3, all the dimensions in the selfreport structure (except stupidity) are bipolar.

The emergence of negative poles in the distinction and depravity dimensions is particularly worth noting. In the case of distinction, the presence of a large low pole denoting mediocrity replicates findings from other self-report studies of evaluative terms where a bipolar Positive Valence dimension emerged (Benet-Martínez and Waller, 1997; although in the studies of Tellegen and Waller, 1987; Almagor et al., 1995, this negative pole is quite modest). The smallish negative pole of depravity, on the other hand, stands out against the monopolarity typically found for this dimension (Negative Valence) in other self-report studies of evaluative terms (Benet-Martínez and Waller, 1997; Tellegen and Waller, 1987; although see Almagor et al., 1995); the emergence of this (smallish) pole, however, is perhaps not surprising given the much larger number of highly evaluative terms used in the present study, relative to most Big Seven studies.

Also noteworthy is the negative pole of unconventionality, which is defined not only by markers of unoriginality (indistinctive, characterless) but also of social decorum (respectable, well-doing). The meaning added by this later descriptor class fits well with the original definition by Tellegen and Waller (1987) of Conventionality in the Big Seven model, a dimension which contrasts a conventional, hyper-socialized disposition (e.g. well-behaved, traditional) against terms denoting a progressive and unconventional life style (e.g. strange, radical).

A final noteworthy difference between the semantic sortings and self-report structures is the lower percentage of total variance accounted for by the self-report factors and their 
lesser degree of simple structure (notice the presence of several cross-loadings at or above 0.40, particularly for worthlessness markers), supporting findings from other studies comparing trait internal and external structures (Peabody and Goldberg, 1989).

When trying to quantitatively measure the similarity of the self-report structure depicted in Table 7 with the internal structures reported in Tables 4 to 6 , standard factor comparison techniques, such as congruence coefficients or multi-sample confirmatory factor analysis, could not be used since the stimuli used across our two studies were not identical. As an alternative, for each factor, we computed the percentage of markers (i.e. terms with loadings equal or higher than 0.40 ) that had primary loadings on similar dimensions in the sorting structures. The percentages were as follows: $89 \%$ for depravity, $85 \%$ for stupidity, $79 \%$ for unconventionality, $76 \%$ for worthlessness, and $72 \%$ for distinction. As these numbers indicate, with the exception of distinction and worthlessness, the overlap is quite high. A close look at the self-report terms with discrepant loadings in the sorting structures reveals that this less than perfect overlap between the sorting and self-report structures is mostly caused by the unique bipolarity of the self-report dimensions (see our previous discussion of the emergent poles for distinction, depravity valence, and unconventionality). ${ }^{13}$

In summary, results from the self-report data largely replicate (with some noteworthy differences) structural findings from study 1 and suggest that dimensions representing distinction, stupidity, unconventionality, worthlessness, and depravity, best organize the actual covariation of the dispositions represented in our sample of highly evaluative personality adjectives, and Norman's (1967) excluded category of 760 evaluative terms more generally.

\section{GENERAL DISCUSSION}

The main goal of this work was to explore the organization and psychological relevance of so-called 'highly evaluative' personality terms (e.g. exceptional, scoundrel, bizarre), a type of personality descriptor that has been historically excluded from the personality taxonomic literature (Allport and Odbert, 1936; Cattell, 1943; Norman, 1967). With that goal in mind, we identified the basic dimensions underlying both semantic-similarity sortings and self-reports, on several representative samples of highly evaluative personality terms.

Four main theoretical issues motivated our research. First, examining the conceptual and self-report structure of highly evaluative personality terms is important given contemporary views that evaluative judgments are at the core of several personality processes (O'Brien and Epstein, 1988; Paulhus and John, 1998), and are uniquely informative about social attributes and behaviours important for human survival (Borkenau, 1990; Buss, 1991; Hogan, 1982; Tellegen, 1993). Second, to the extent that traditional lexical models of personality (e.g. the Big Five) have systematically excluded highly evaluative terms, it is important to explore how much these expressions inform us

\footnotetext{
${ }^{13}$ One possible reason behind the monopolarity of the sorting structures is that not enough antonym piles were generated by our participants in the semantic sorting exercise for bipolar definitions of the factors to emerge; if true, this finding would suggest perhaps an interesting form of 'cognitive economy', one that makes conceptualizing opposite-meaning attributes (e.g. topnotch and unsatisfactory) as two unrelated constructs (i.e. putting them in two separate piles) 'easier', that is, less cognitively effortful, than integrating them into a single (but complex) oppositional construct, despite the fact that, in actuality, these two attributes probably covary (negatively) in people.
} 
about psychological domains perhaps not well represented in these models (although note that our exploratory study does not directly address this issue). Third, examining the selfreport structure of highly evaluative personality terms can help clarify and expand the conceptual scope of existing evaluative constructs such as Positive and Negative Valence (Tellegen and Waller, 1987) and the 'superhero' and 'sainthood' complexes (Paulhus and John, 1998). Lastly, numerous studies have examined and compared self-report and semantic-similarity sorting structures for traits and affects (e.g. Church and Katigbak, 1989; Russell, 1978; Watson, Clark and Tellegen, 1984), but this issue had yet to be addressed in the domain of evaluation.

In study 1 we examined the structure of semantic-similarity sortings collected on three sets of adjectives randomly selected from Norman's (1967) excluded category of 'evaluative' personality terms. We were able to identify a set of five dimensionsdistinction, worthlessness, depravity, stupidity, and unconventionality — that was common to all three sets.

In study 2 we examined the structure of self-reports collected on a larger sample of highly evaluative personality descriptors that combined the terms used in study 1 . Analyses yielded a five-factor structure with dimensions conceptually similar to those uncovered in the sorting data. One significant difference between the sorting and selfreport structures was that most of the self-report dimensions were clearly bipolar. As mentioned earlier, this difference is probably rooted in a combination of psychometric and conceptual factors. First, study 2's reliance on a much larger sample of terms is probably linked to the broader factor definitions obtained in the self-report structure. Second, the cognitive economy typically associated with semantic-similarity sortings (Peabody and Goldberg, 1989) may have influenced participants' ability to classify opposite meaning terms into antonym piles, leading to fewer bipolar factor definitions in the sorting data. Lastly, the ipsatization of the self-report data may have emphasized the bipolarity of the self-report dimensions (see Hamilton, 1968, for a discussion of how ipsatization helps to control for response bias, a type of influence that often obscures the true bipolarity of dimensions). Despite these differences, the overlap between the external and internal structures is quite high, as indicated by the percentage of self-report and sorting factor markers with primary loadings on similar dimensions. Next we discuss the implications of this overlap for the ontological status of highly evaluative personality terms.

\section{Overlap between internal and external structures}

In the introduction we discussed several arguments proposed to explain the overlap between external and internal structures for traits and affects. A favored view argues that overlap occurs because similarity judgments reflect not only people's conceptual understanding of the particular attributes but also people's own memory of how these attributes covary in their lives (Borkenau, 1992; Schimmack and Reisenzein, 1997). In the context of our studies, should we also have expected a high degree of similarity between external and internal structures for highly evaluative personality descriptors?

Recall the contention made by researchers who excluded 'evaluative' descriptors in their taxonomic studies (Allport and Odbert, 1936; Cattell, 1943; Norman, 1967), that is, that these descriptors are too ambiguous and void of descriptive components to be behaviourally informative. If this view were valid, then one would expect the overlap between internal and external structures for these terms to be low. This argument works as follows: if highly evaluative personality descriptors are too extreme and ambiguous to 
capture reliable conceptual, personality-relevant variance, then self-reports on these descriptors should mostly represent response bias and yield two 'purely' evaluative dimensions separating desirable and undesirable terms, rather than yielding content personality dimensions). ${ }^{14}$ Relative to self-reports, however, semantic-similarity sortings on these terms should be more likely to yield meaningful (i.e. content-relevant) dimensions, given that similarity sortings are clearly guided by objective meaning assessment rather than by protective self-description (as in the self-reports).

Clearly, results from our studies do not support this argument. Structures obtained from self-reports and semantic-similarity sortings were quite similar at the structural and conceptual level. This finding suggests that, regardless of the rating methodology used, the conceptual component of so-called 'highly evaluative' descriptors accounts for more variance than their purely evaluative constituent. In short, one conclusion that warrants consideration is that typically excluded highly evaluative personality terms, far from being behaviourally ambiguous and psychologically uninformative, allude to meaningful dispositions that people both implicitly understand and possess to different degrees. In the next section we discuss the meaning of these dispositions and their relevance to personality description.

\section{How relevant to personality are the uncovered dimensions?}

Results from our studies of highly evaluative personality descriptors indicate that variance from both semantic-similarity sortings and self-reports is organized along five dimensions representing distinction, worthlessness, depravity, unconventionality, and stupidity. Notice that these dimensions (except stupidity) capture the three evaluative elements that are unique to the Big Seven (Tellegen and Waller, 1987), namely, Positive Valence, Negative Valence, and the evaluative component of Openness to Experience (i.e. perceptions of conventionality-eccentricity). Positive Valence, or its subcomponents, is represented in our studies by the two dimensions we named distinction and worthlessness. A dimension resembling Negative Valence (depravity) was clearly represented in our data. The Big Seven's conventionality-eccentricity component is captured by our unconventionality dimension. These conceptual correspondences are noteworthy given the independence between traditional Big Seven studies - which typically rely on samples of dictionarybased terms that include many Big Five markers - and our two studies, which relied solely on terms from Norman's excluded 'evaluative' category. These findings also suggest that personality evaluative judgments are organized around the basic domains of morality (i.e. depravity), power (distinction and worthlessness), peculiarity (unconventionality), and intelligence (stupidity).

The particular relevance to personality research of the above evaluative domains (and of highly evaluative personality terms in general) deserves some discussion. As suggested by a reviewer, obtaining self- or other-ratings on the evaluative domains of morality, power, peculiarity, and intelligence may prove particularly useful in the following cases: (1) in

\footnotetext{
${ }^{14}$ Notice that the Positive and Negative Valence dimensions reported in Big Seven studies (Almagor et al., 1995; Benet-Martínez and Waller, 1997; Tellegen and Waller, 1987) are not simply two 'purely' evaluative (i.e. meaning-empty) dimensions separating desirable and undesirable terms. In these studies, Positive and Negative Valence are each defined by clusters of terms which share unique descriptive variance (one dimension denotes power and the other morality), rather than just desirability or undesirability. In fact, in some of these studies (Almagor et al., 1995; Benet-Martínez and Waller, 1997), Positive and Negative Valence emerge as separate bipolar dimensions each containing a combination of desirable and undesirable terms. Furthermore, most Big Seven studies have relied on a limited number of evaluative terms which may explain why only two independent evaluative dimensions were uncovered.
} 
professional settings where anonymous person descriptions are needed (e.g. instructional or managerial evaluations), (2) in clinical settings where honest self-disclosure is desirable; (3) when coding free person descriptions such as those found in biographies, diaries, legal proceedings, testimonials, political campaigns, etc. Furthermore, ratings on highly evaluative personality descriptions may prove useful in understanding cultural differences in natural personality judgments (e.g. work on parental child personality descriptions by Kohnstamm, Halverson, Mervielde, and Havill, 1998, indicates differential cultural sensitivities to the use of negative terms). Next we discuss each of the five evaluative dimensions obtained in our study in terms of its meaning and heuristic value.

The emergence of two separate factors tapping different components of Positive Valence is particularly noteworthy. Why did our highly evaluative terms define distinction and worthlessness factors instead of forming a single Positive Valence dimension? Methodologically, this is perhaps not surprising given that all Big Seven studies (e.g. Almagor et al., 1995; Benet-Martínez and Waller, 1997; Tellegen and Waller, 1987) have relied on smaller pools of evaluative descriptors. Psychologically, on the other hand, the differentiation between distinction and worth seems meaningful and in accordance with the recent theoretical differentiation between 'self-competence' and 'self-liking' components of self-esteem (Tafarodi and Swann, 1995). ${ }^{15}$ Self-competence and selfliking, like distinction and worthlessness in our studies, differentiate between the successor competence-related components of esteem and those related to personal worth. The psychological relevance and psychometric independence of distinction and worthlessness is further supported by recent evidence showing that self-competence and self-liking, despite being often highly associated, have different psycho-developmental and cultural correlates (Tafarodi and Swann, 1995, 1996).

Consider our depravity findings. This dimension underscores an element of person description that is receiving renewed attention: the moral dimension of personality (Bandura, 1999; Cawley, Martin and Johnson, 2000). Notice that depravity markers (e.g. evildoer, damnable, rat versus unmarred, unstained, undepraved) relate to individuals' judgments of malice-virtue, a type of person evaluation that recent socio-evolutionary views of morality (Kirkpatrick, 1999), shame, guilt (D'Arms, unpublished dissertation), and evil (Baumeister and Campbell, 1999), describe as an universal perceptual mechanism that is basic for survival. Given the recent evidence showing that inflated, overpositive selfviews, when combined with an ego threat, often lead to immoral and violent behaviour (Baumeister, Smart and Boden, 1999), future research should examine the relation between high levels of self-reported distinction and (low) worthlessness and observerreports of depravity.

The robustness of depravity in our studies also speaks to the scope of esteem-related processes by underscoring the role that morality perceptions play in evaluating people. This would suggest that traditional models and measures of self-esteem (which typically define esteem in terms of competence and/or worth solely) might benefit from incorporating perceptions of morality in their theories. The significance of moral perceptions in evaluating people is further supported by person perception research showing that people attend to and weight morality information more than competence

\footnotetext{
${ }^{15}$ Examples of the items of Tafarodi and Swann measuring 'self-liking' are 'I am secure in my sense of selfworth,' and (reverse) 'I feel worthless at times'. Examples of 'self-competence' items are 'I perform very well at a number of things' and 'I am talented'.
} 
information when forming impressions and expectations about others (De Bruin and Van Lange, 2000; Wojciszke, Bazinska and Jaworski, 1998).

With regard to the psychological relevance of unconventionality, we believe that the eccentricity-conventionality continuum defined by our highly evaluative terms (e.g. odd, bizarre, peculiar versus indistinctive, characterless, respectable) broadens and clarifies the psycho-lexical boundaries of the Big Five's Openness to Experience dimension. This view is clearly spelled out by Waller (1999) in his interpretation of the Big Seven's Conventionality dimension: 'People who are relatively "closed" to new experiences describe themselves in conventional terms; they espouse traditional values and beliefs and have natural respect for authority and rules. Individuals who are "open" to their environment... describe themselves as being progressive and radical. Being excessively open has its dangers, however... other less open individuals may describe you as being odd, strange, and peculiar' (p. 19). One way to clarify and establish the meaning and predictive validity of unconventionality would be to study the relation between this construct and clinical observations of the 'eccentric personality' (Week and Turner, 1996). Specifically, unconventionality may prove useful in validating the observations by Week and Turner that eccentric people are extremely high in Openness traits such as absorption and novelty seeking, have positive mental and physical health (contrary to popular belief), but also frequently experience feelings of 'marginality'.

Finally, the emergence of a stupidity dimension, and its stability across sorting sets and across internal and external structures, is also worth discussing. Recall that this dimension has never emerged in other lexical studies using pools of terms that included evaluative descriptors (Benet-Martínez and Waller, 1997; Tellegen and Waller, 1987). Given that these lexical studies included few (or zero) markers of low intelligence (e.g. see idiotic in Table 1 from Benet-Martínez and Waller, 1997), the presence of a stupidity dimension in our data is interesting but not surprising. In fact, a close examination of the structure reported in the original study by Tellegen and Waller (1987) reveals the presence of only six terms denoting low intelligence (stupid, pinheaded, thick, oafish, chuckleheaded, dense), all loading on the Negative Valence factor. Conceptually, on the other hand, our stupidity dimension captures evaluative judgments of cognitive abilities, a domain that most psychologists believe falls outside the focus of personality psychology (McCrae and Costa, 1985; although see indirect measures of intelligence in the personality measures of Gough, 1996; Hogan and Hogan, 1992). Stupidity, therefore, unlike the other four dimensions uncovered in our studies, may not prove as informative regarding personalityrelevant evaluative processes, unless one is interested in 'neurotic stupidity', an obscure psychodynamic construct used to describe the defensive self-ascribed feelings of intellectual incompetence often associated with 'infantile personality' (Oberndorf, 1939; Van Bark, 1954).

Another prominent feature in the structures uncovered in our studies is the overrepresentation of negative (i.e. undesirable) terms over positive ones. An inspection of Table 7 reveals that 135 terms, out of 202, are negative (a ratio of 67\%). The predominance of negative descriptors in our samples of highly evaluative adjectives (and Norman's evaluative category) is potentially informative about specific socio-cognitive processes involved in personality perception. Specifically, it suggests that verbalized evaluations of human character and behaviour are disproportionally negative. One possible reason for this is that perceptions of undesirable human attributes (e.g. being immoral), relative to desirable ones (e.g. being moral), are more powerful in eliciting affective judgments (i.e. evaluations) from people. This interpretation would be in accordance with 
the 'negativity bias' in affective processing reported in the social-cognition literature (Fiske, 1980; Ito, Larsen, Smith and Cacioppo, 1998; Wojciszke et al., 1993; but see Martijn, Spears, Van der Pligt and Jakobs, 1992). At the same time, the predominance of negative descriptors in our dimensions may reflect possible overlooked nuances in Norman's (1967) exclusionary decisions. Specifically, it suggests that in 'evaluating' what an 'evaluative' personality descriptor is, Norman may have favoured negative terms over desirable ones.

Given the primarily exploratory nature of our studies, our findings are inconclusive with regard to at least two important issues. First and most importantly, because we did not explore the overlap/specificity between our dimensions and the Big Five, we cannot yet draw definite conclusions about the degree to which distinction, worthlessness, depravity, and unconventionality dimensions inform about possible domains of personality neglected in traditional models of personality. Future studies should explore this issue in the context of not only the Big Five but also other relevant personality constructs such as narcissism, self-esteem, and morality (Benet-Martínez, manuscript in preparation). Second, the cultural, linguistic, and perceptual generalizability of our findings is limited by the fact that our studies relied exclusively on one language (English) and on one type of target (self-reports). Future studies should examine the organization and psychological relevance of highly evaluative personality terms in other languages and cultures and on other types of target such as peer-reports or reports of strangers.

\section{Conclusion}

The two studies reported in this paper have shown that typically excluded English highly evaluative personality descriptors, far from being behaviourally ambiguous and psychologically uninformative, allude to meaningful dispositions that people implicitly understand and posses to different degrees. The dispositions identified in our studiesdistinction and worthlessness (two facets of Positive Valence), depravity, unconventionality, and, to a lesser extent, stupidity (for reasons outlined earlier) — are informative regarding the conceptual scope of the Big Seven factor model. Furthermore, these dimensions may underscore meaningful personality variance that has been overlooked in traditional personality models. This claim, however, needs to be tested by future studies where the statistical independence/overlap between our dimensions and traditional personality dispositions, such as those represented in the Big Five, is examined. Similarly, future research should examine the value of distinction, worthlessness, depravity, and unconventionality to predict important self-esteem related outcomes, and the association between these dimensions and self-perception biases (Paulhus and John, 1998) and multidimensional measures of self-esteem (O'Brien and Epstein, 1988; Tafarodi and Swann, 1995).

In conclusion, we believe that our findings attest to the idea that evaluative judgments, even extreme ones, are an integral component of human personality description.

\section{ACKNOWLEDGEMENT}

This research was supported in part by grants from the University of Michigan's Rackham School of Graduate Studies and the Spanish Ministry of Science and Education (Ministerio de Educación y Ciencia) to the first author. We are very grateful to Auke Tellegen for his methodological assistance with the analyses of the sorting data, to Samuel Gosling for his 
prompt and helpful comments on an earlier version of this manuscript, and to several anonymous reviewers who read an earlier version of this paper. We also thank Mark Akiyama, Osmara Reyes, Patricia Altemura, Rachna Rajan, Maria Magallanes, and Anne Choi for their assistance with the data collection and entry, and Greg Spooner for his help labelling the factorial dimensions.

\section{REFERENCES}

Allport GW, Odbert HS. 1936. Trait names: a psycho-lexical study. Psychological Monographs 47(211).

Almagor M, Tellegen A, Waller NG. 1995. The Big Seven Model: a cross-cultural replication and further explorations of the basic dimensions of natural language trait descriptors. Journal of Personality and Social Psychology 69: 300-307.

Angleitner A, Ostendorf F, John OP. 1990. Towards a taxonomy of personality descriptors in German: a psycho-lexical study. European Journal of Personality 4: 89-118.

Baer RA, Kroll LS, Rinaldo J, Ballenger J. 1999. Detecting and discriminating between random responding and overreporting on the MMPI-A. Journal of Personality Assessment 72: 308-320.

Bandura A. 1999. Moral disengagement in the perpetration of inhumanities. Personality and Social Psychology Review 3(Special Issue: Perspectives on Evil and Violence): 193-209.

Baumeister RF, Campbell WK. 1999. The intrinsic appeal of evil: sadism, sensational thrills, and threatened egotism. Personality and Social Psychology Review 3(Special Issue: Perspectives on Evil and Violence): 210-221.

Baumeister RF, Smart L, Boden JM. 1999. Relation of threatened egotism to violence and aggression: the dark side of high self-esteem. In The Self in Social Psychology, Baumeister RF (ed.). (pp. 240-284). Psychology Press-Taylor Francis: Philadelphia, PA.

Benet-Martínez V. 1999. Exploring indigenous Spanish personality constructs with a combined emic-etic approach. In Latest Contributions to Cross-Cultural Psychology, Lasry JC, Adair JG, Dion KL (eds). (pp.). Swets and Zeitlinger: Lisse.

Benet-Martínez V, John OP. 1998. 'Los Cinco Grandes' across cultures and ethnic groups: multitrait-multimethod analyses of the Big Five in Spanish and English. Journal of Personality and Social Psychology 75: 729-750.

Benet V, Waller NG. 1995. The 'Big Seven' model of personality description: evidence for its crosscultural generality in a Spanish sample. Journal of Personality and Social Psychology 69: 701-718.

Benet-Martínez V, Waller NG. 1996, June. Positive and Negative Valence: Meaningful Lexical Dimensions of Personality Description Outside the Big Five. Poster presented at the Annual Convention of the American Psychological Society, San Francisco.

Benet-Martínez V, Waller NG. 1997. Further evidence for the cross-cultural generality of the 'Big Seven' model: imported and indigenous Spanish personality constructs. Journal of Personality 65: 567-598.

Block J, Weiss DS, Thorne A. 1979. How relevant is a semantic similarity interpretation of personality ratings? Journal of Personality and Social Psychology 37: 1055-1074.

Blumberg D. 1973. Description, evaluation, and obligation. Mind 82: 348-357.

Borkenau P. 1990. Traits as ideal-based and goal-derived social categories. Journal of Personality and Social Psychology 58: 381-396.

Borkenau P. 1992. Implicit personality theory and the five-factor model. Journal of Personality 60: 295-327.

Brewer MB, Lui LN. 1996. Use of sorting tasks to assess cognitive structures. In Answering questions: Methodology for Determining Cognitive and Communicative Processes in Survey Research, Schwarz N, Sudman S (eds). Jossey-Bass: San Francisco, CA.

Bromley DB. 1977. Personality Description in Ordinary Language. Wiley: New York.

Burger LK, Miller PJ. 1999. Early talk about the past revisited: affect in working-class and middleclass children's co-narrations. Journal of Child Language 26: 133-162.

Buss DM. 1991. Evolutionary personality psychology. Annual Review of Psychology 42: 459-491. 
Cacioppo JT, Gardner WL, Berntson GG. 1997. The affect system has parallel and integrative processing components: form follows function. Journal of Personality and Social Psychology 76: 839-855.

Cattell RB. 1943. The description of personality: basic traits re-solved into clusters. Journal of Abnormal and Social Psychology 38: 476-506.

Cawley MJ, Martin JE, Johnson JA. 2000. A virtues approach to personality. Personality and Individual Differences 28: 997-1013.

Church AT, Katigbak MS. 1989. Internal, external, and self-report structure of personality in a nonWestern culture. An investigation of cross-language and cross-cultural generalizability. Journal of Personality and Social Psychology 57: 857-872.

Church AT, Katigbak MS, Reyes JA. 1998. Further exploration of Filipino personality structure using the lexical approach: do the Big-Five or Big-Seven dimensions emerge? European Journal of Personality 12: 249-269.

Church AT, Reyes JA, Katigbak MS, Grimm SD. 1997. Filipino personality structure and the Big Five model: a lexical approach. Journal of Personality 65: 477-528.

D'Andrade RG. 1985. Character terms and cultural models. In Directions in Cognitive Anthropology, Dougherty WD (ed.). (pp. 321-343). University of Illinois Press: Urbana, IL.

De Bruin EN, Van Lange PA. 2000. What people look for in others: influences of the perceiver and the perceived on information selection. Personality and Social Psychology Bulletin 26: 206-219.

De Raad B, Hoskens M. 1990. Personality descriptive nouns. European Journal of Personality 4: 89-115.

De Raad B, Mulder E, Kloosterman K, Hofstee W. 1988. Personality-descriptive verbs. European Journal of Personality 2(Special Issue: Personality Measurement): 81-96.

Di Blas L, Forzi M, Peabody D. 2000. Evaluative and descriptive dimensions from Italian personality factors. European Journal of Personality 14: 279-290.

Emmons RA. 1997. Motives and goals. In Handbook of Personality Psychology, Hogan R, Johnson JA, Briggs SR (eds). (pp. 485-512). Academic: San Diego, CA.

Fiske ST. 1980. Attention and weight in person perception: the impact of negative and extreme behavior. Journal of Personality and Social Psychology 38: 889-906

Giammatteo M. 1998. La dimension expresivo-evaluativa en entrevistas orales con estudiantes universitarios (The expressive-evaluative dimension in oral interviews with university students). Estudios de Linguistica 12: 79-92.

Goldberg LR. 1982. From Ace to Zombie: some explorations in the language of personality. Advances in Personality Assessment 1: 203-234.

Goldberg LR. 1992. The development of markers for the Big-Five factor structure. Psychological Assessment: A Journal of Consulting and Clinical Psychology 4: 26-42.

Goldberg LR. 1993. The structure of phenotypic personality traits. American Psychologist 48: $26-34$.

Gough HG. 1996. The California Psychological Inventory (3rd ed.). Consulting Psychologists: Palo Alto, CA.

Guadagnoli E, Velicer WF. 1988. Relation to sample size to the stability of component patterns. Psychological Bulletin 103: 265-275.

Hamilton DL. 1968. Personality attributes associated with extreme response style. Psychological Bulletin 69: 192-203.

Harkness AR. 1992. Fundamental topics in personality disorders: candidate trait dimensions from lower regions of the hierarchy. Psychological Assessment 4: 251-259.

Hassebrauck M, Thomas B. 1996. Three-dimensional love. Journal of Social Psychology 136: $121-122$.

Hoff-Ginsberg E. 1997. Frog stories from four-year-olds: individual differences in the expression of referential and evaluative content. Journal of Narrative and Life History 7: 223-227.

Hogan R. 1982. A socio-analytic theory of personality. In Nebraska Symposium on Motivation, 1982: Personality-Current Theory and Research Lincoln, Page MM (ed.). University of Nebraska Press: NE.

Hogan R. 1996. A socioanalytic perspective on the Five-Factor Model. In The Five-Factor Model of Personality: Theoretical Perspectives, Wiggins JS (ed.). (pp. 163-180). Guilford: New York.

Hogan R, Hogan J. 1992. Hogan Personality Inventory Manual. Hogan Assessment Systems: Tulsa, OK. 
Hyman IE. 1994. Conversational remembering: story recall with peer versus for an experimenter. Applied Cognitive Psychology 8: 49-66.

Ito TA, Larsen JT, Smith NK, Cacioppo JT. 1998. Negative information weighs more heavily on the brain: the negativity bias in evaluative categorizations. Journal of Personality and Social Psychology 75: 887-900.

Jauregui B. 1973. Clasificacion de los adjetivos atributivos en castellano desde el punto de vista sicolinguistico (Classification of attributive adjectives in Castilian from the psycholinguistic point of view). Lenguaje y Ciencias 13: 12-18.

John OP. 1990. The 'Big Five' factor taxonomy: dimensions of personality in the natural language and in questionnaires. In Handbook of Personality: Theory and Research, Pervin LA (ed.). (pp. 66-100). Guilford: New York.

John OP, Angleitner A, Ostendorf F. 1988. The lexical approach to personality: a historical review of trait taxonomic research. European Journal of Personality 2: 171-203.

John OP, Robins RW. 1993. Gordon Allport: father and critic of the Five-Factor Model. In Fifty Years of Personality Psychology, Craik KH, Hogan R, Wolfe RN (eds). (pp. 215-236). Plenum: New York.

Kaplan MT. 1973. Evaluative judgments are based on evaluative information: evidence against meaning change in evaluative context effects. Memory and Cognition 3: 375-380.

Kim MP, Rosenberg S. 1980. Comparison of two structural models of implicit personality theory. Journal of Personality and Social Psychology 38: 375-389.

Kirkpatrick LA. 1999. Toward and evolutionary psychology of religion and personality. Journal of Personality 67(Special Issue: Religion in the Psychology of Personality): 921-952.

Kohnstamm GA, Halverson CF, Mervielde I, Havill VL. 1998. Parental Descriptions of Child Personality: Developmental Antecedents of the Big Five? Erlbaum: Mahwah, NJ.

Kuusinen J. 1969. Affective and denotative structures of personality ratings. Journal of Personality and Social Psychology 12: 181-188.

Larsen RJ, Diener E. 1992. Promises and problems with the circumplex model of emotion. In Review of Personality and Social Psychology (Vol. 13, pp. 25-59), Clark MS (ed.). Sage: Newbury Park, CA.

Lewicka M. 1979. Effect of cognitive set—to diagnose or to evaluate-upon impression formation and change. Polish Psychological Bulletin 10: 21-30.

Marquez E. 1998. Classification des adjectifs: etude exploratoire sur l'organisation semantiquepragmatique des adjectifs (Adjective classification: exploratory study of the semantic-pragmatic organization of adjectives). Langages 32: 87-107.

Martijn C, Spears R, Van der Pligt J, Jakobs E. 1992. Negativity and positivity effects in person perception and inference: ability versus morality. European Journal of Social Psychology 22(Special Issue: Positive-Negative Asymmetry in Affect and Evaluations): 453-463.

Maslow AH. 1970. Motivation and Personality (2nd ed.). Harper and Row: New York.

McCrae RR, Costa PT. 1985. Updating Norman's 'adequacy taxonomy': intelligence and personality dimensions in natural language and in questionnaires. Journal of Personality and Social Psychology 49: 710-721.

McCrae RR, Costa PT. 1995. Positive and negative valence within the five-factor model. Journal of Research in Personality 29: 443-460.

McCrae RR, Costa PT. 1997. Personality trait structure as a human universal. American Psychologist 52: $509-516$.

Mervielde I. 1994. A five-factor model classification of teachers' constructs on individual differences among children ages 4 to 12. In The Developing Structure of Temperament and Personality from Infancy to Adulthood, Havelson CF, Kohnstamm GA, Martin RP (eds). (pp. 387-397). Erlbaum: Hillsdale, NJ.

Norman WT. 1967. 2800 Personality Trait Descriptors: Normative Operating Characteristics for a University Population. University of Michigan Department of Psychology: Ann Arbor, MI.

O'Brien EJ, Epstein S. 1988. MSEI: The Multidimensional Self-Esteem Inventory. Psychological Assessment Resources: Odessa, FL.

Oberndorf CP. 1939. The feeling of stupidity. International Journal of Psycho-Analysis 20: 443-451.

Osgood CE, Suci GJ. 1955. Factor analysis of meaning. Journal of Experimental Psychology 50: 325-338. 
Paulhus DL, John OP. 1998. Egoistic and moralistic biases in self-perception: the interplay of selfdeceptive styles with basic traits and motives. Journal of Personality 66: 1025-1060.

Paunonen SV, Jackson DN. 2000. What is beyond the Big Five? Plenty! Journal of Personality 68: 821-835.

Peabody D. 1984. Personality dimensions through trait inferences. Journal of Personality and Social Psychology 46: 384-403.

Peabody D, Goldberg LR. 1989. Some determinants of factor structures from personality-trait descriptors. Journal of Personality and Social Psychology 57: 552-567.

Pervin LA. 1983. The stasis and flow of behavior: toward a theory of goals. In Nebraska Symposium on Motivation, Page MM (ed.). (pp. 1-53). University of Nebraska Press: Lincoln, NE.

Reisenzein R, Schimmack U. 1999. Similarity judgments and covariation of affects: findings and implications for affect structure research. Personality and Social Psychology Bulletin 5: $539-555$.

Rivière C. 1983. Modal adjectives: transformations, synonymy, and complementation. Lingua 59: 145.

Roid GH, Fitts WH. 1988. Tennessee Self-Concept Scale (revised manual). Western Psychological Services: Los Angeles.

Rosenberg S, Sedlak A. 1972. Structural representation of perceived personality trait relationships. In Multidimensional scaling: Theory and Applications in the Behavioral Sciences, Shepard R, Romney K, Nerlove S (eds). Academic: New York.

Russell JA. 1978. Evidence and convergent validity on the dimensions of affect. Journal of Personality and Social Psychology 36: 1152-1168.

Russell JA, Barrett LF. 1999. Core affect, prototypical emotional episodes, and other things called emotion: dissecting the elephant. Journal of Personality and Social Psychology 76: 805-819.

Saucier G. 1994a. August. Positive and Negative Valence: Beyond the Big Five? Paper presented at the Annual Convention of the American Psychological Association, Los Angeles, CA.

Saucier G. 1994b. Separating description and evaluation in the structure of personality attributes. Journal of Personality and Social Psychology 66: 141-154.

Saucier G. 1997. Effect of variable selection on the factor structure of person descriptors. Journal of Personality and Social Psychology 73: 1296-1312.

Saucier G, Goldberg LR. 1998. What is beyond the Big Five? Journal of Personality 66: 495-524.

Schimmack U, Reisenzein R. 1997. Cognitive processes involved in similarity judgments of emotions. Journal of Personality and Social Psychology 73: 645-661.

Schmidt PF. 1984. Self-esteem: assessing the moral dimension. Journal of Psychology and Christianity 3: 52-56.

Shweder RA, D'Andrade RG. 1980. The systematic distortion hypothesis. In Fallible Judgement in Behavioral Research: New Directions for the Methodology of Social and Behavioral Science (Vol. 4. pp. 37-58), Shweder RA (ed.). Jossey-Bass: San Francisco.

Simon-Vandenbergen AM. 1995. Assessing linguistic behaviour: a study of value judgements. In By Word of Mouth: Metaphor, Metonymy and Linguistic Action in a Cognitive Perspective, Goossens L (ed.). Benjamins: Amsterdam.

Sneed CD, McCrae RR, Funder DC. 1998. Lay conceptions of the five-factor model and its indicators. Personality and Social Psychology Bulletin 24: 115-126.

Sternberg RJ. 1988. Triangulating love. In The Psychology of Love, Sternberg RJ, Barnes M (eds). Yale University Press: New Haven, CT.

Tafarodi RW, Swann WB. 1995. Self-liking and self-competence as dimensions of global selfesteem: initial validation of a measure. Journal of Personality Assessment 65: 322-342.

Tafarodi RW, Swann WB. 1996. Individualism-collectivism and global self-esteem: evidence for a cultural trade-off. Journal of Cross-Cultural Psychology 27: 651-672.

Tellegen A. 1993. Folk concepts and psychological concepts of personality and personality disorder. Psychological Inquiry 4: 122-130.

Tellegen A, Waller NG. 1987. Reexamining Basic Dimensions of Natural Language Trait Descriptors. Paper presented at the annual meeting of the American Psychological Association, New York.

Tellegen A, Waller NG. in press. Exploring personality through test construction: development of the Multidimensional Personality Questionnaire. In Personality Measures: Development and Evaluation (Vol. 1), Briggs SR, Cheek JM (eds). JAI Press: Greenwich, CN. 
Tesser A, Martin L. 1996. The psychology of evaluation. In Social Psychology: Handbook of Basic Principles, Higgins T, Kruglanski A (eds). (p. 400-432). Guilford Press: New York.

Van Bark BS. 1951. Smartness and stupidity in neurosis. American Journal of Psychoanalysis 11: $36-41$.

Van der Pligt J, Taylor C. 1984. Trait attribution: evaluation, description, and attitude extremity. European Journal of Social Psychology 14: 211-221.

Waller NG. 1999. Evaluating the structure of personality. In Personality and Psychopathology, Cloninger CR (ed.). American Psychiatric Press: Washington, DC.

Waller NG, Lykken D, Tellegen A. 1995. Occupational interests, leisure time interests, and personality: three domains or one? Findings from the Minnesota Twin Registry. In Assessing Individual Differences in Human Behavior: New Concepts, Methods, and Findings, Lubinski DJ, Dawis RV (eds). (p. 233-259). Davies-Black: Palo Alto, CA.

Watson D, Clark LA, Tellegen, A. 1984. Cross-cultural convergence in the structure of mood: a Japanese replication and a comparison with the U.S. findings. Journal of Personality and Social Psychology 47: 127-144.

Watson D, Clark LA, Tellegen A. 1988. Development and validation of brief measures of Positive and Negative Affect: the PANAS scales. Journal of Personality and Social Psychology 54: 10631070.

Watson D, Wiese D, Vaidya J, Tellegen A. 1999. The two general activation systems of affect: structural findings, evolutionary considerations, and psychobiological evidence. Journal of Personality and Social Psychology 76: 820-838.

Week DJ, Turner P. 1996. Eccentrics: A Study of Sanity and Strangeness. Kodansha: New York.

Wiggins JS. 1964. Convergences among stylistic response measures from objective personality tests. Psychological Measurement 24: 551-562.

Wiggins JS. 1973. Personality and Prediction: Principles of Personality Assessment. AddisonWesley: Reading, MA.

Wojciszke B, Bazinska R, Jaworski M. 1998. On the dominance of moral categories in impression formation. Personality and Social Psychology Bulletin 24: 1251-1263.

Wojciszke B, Brycz H, Borkenau P. 1993. Effects of information content and evaluative extremity on positivity and negativity biases. Journal of Personality and Social Psychology 64: 327-335.

Yang K, Bond MH. 1990. Exploring implicit personality theories with indigenous or imported constructs: the Chinese case. Journal of Personality and Social Psychology 58: 1087-1095. 


\section{APPENDIX: THREE SETS OF HIGHLY EVALUATIVE TERMS RANDOMLY SELECTED FROM NORMAN'S (1967) EXCLUSION CATEGORY 1.2}

\section{Set 1}

ACCEPTABLE; Allowable; Satisfactory

ADORABLE; Delightful; Charming; Lovely

ARRANT; Notoriously bad

ASSISH; Resembling an ass, Stupid; Obstinate

BALANCED; Emotionally stable

BALMY; Mildly insane; Eccentric

BASE-MINDED; Morally low-minded

BLOCKHEADED; Stupid; Unintelligent

BLUNDERHEAD; Stupid; Ignorant

BOUNDER; A vulgar person of obtrusive manners

BUZZARD; Rapacious; Avaricious person

CIPHER; Insignificant; Worthless

CONSIDERABLE; Important; Significant

CONTEMPTIBLE; Worthy of contempt; Deserving disdain

CRACKBRAINED; Crazy; Reasonless

DEMIGOD; One so prominent in intellect, power, ability, beneficence, or appearance as to seem to approach the divine

DENIGRATED; Dishonoured; Defamed

DISAGREEABLE; Ill-tempered; Irritable

DISGRADED; Disgraced; Abased

ENGRACED; Favoured; Brought into favour

EXCELLED; One who surpasses others or is superior in some respect

FALLEN; Degraded or immoral, having lost one's chastity

FALLIBLE; Liable to err

FAULTLESS; Without fault; Free from defect

FIRST-CLASS; Of the highest grade; Excellent

FOOLISH; Repulsive; Repellent

GOONEY; Idiot

HALF-WITTED; Foolish; Stupid

HIGH-CALIBRE; Of high degree of excellence

HONOURLESS; Not of dignity; Not of respect

INESSENTIAL; Not necessary; Needless

INEXPERT; Inexperienced; Not skilled

LOUSY; Contemptible; Disgusting

LOUTISH; Awkward; Clumsy; Boorish

LUMPISH; Sluggish; Dull; Clumsy

LUNKHEAD; Blockhead; Imbecile

MIGHT-HAVE-BEEN; A person who might have amounted to something

NIFTY; Attractively smart or stylish; Fine

NONESSENTIAL; Not of prime or central importance; Dispensable

NULL; Of no efficacy; Invalid

OFF; Away from what is considered normal, regular, or standard

OUTRE; Extravagant; Bizarre 
OVERGREAT; Above and beyond great; Markedly superior

OVERLUSCIOUS; Having excessive sensual appeal

PARALLELLESS; Unmatched; Without equal

PIDDLING; Trifling; Trivial

PLAIN; Not intricate; Simple

PLUPERFECT; More than perfect

POINTLESS; Without meaning or relevance

PROFICIENT; Well advanced in any occupation or knowledge

PUKISH; Offensive

RAT; Scoundrel; Villain

RESPECTWORTHY; Worthy of respect (honor)

SCOUNDREL; Dishonorable man; Villain

SCUMMY; Low class; Worthless

STAINLESS; Spotless; Pure

STELLAR; Of brilliance; Outstanding

SUBLIMISH; Magnificent; Superb

SUPEREMINENT; Extremely distinguished

THIRD-CLASS; Pertaining to a class or rank next below the second

UNCAPABLE; Incapable; Unable

UNCOMMON; Not ordinarily encountered; Unusual

UNCONSEQUENTIAL; Inconsequential; Unimportant

UNEXCELLENT; Not of high quality

UNEXEMPLARY; Not commendable; Not meritorious

UNFLAWED; One who has no shortcomings

UNGENUINE; Not real; Fake

UNHARMFUL; Not able to cause harm

UNIDEAL; Not a model of excellence; Not exemplar

UNMARRED; Not defaced; Not blemished

UNNECESSARY; Not necessary; Needless

UNPARALLELLABLE; Having no equal; Unmatchable

UNSMIRCHED; Not dishonored or defamed

WELL-DOING; Kind-hearted; Diligent

WORTHLESS; Lacking merit or worth

\section{Set 2}

BEASTLY; Nasty; Disagreeable

BEEF-WITTED; Stupid; Dull

BIZARRE; Odd; Extravagant

BLOCKHEADED; Stupid; Unintelligent

BLOT; Disgrace; Blemish

BUNGLESOME; Inept; Inefficient

BUZZARD; Rapacious; Avaricious person

CAD; With ungentlemanly manners

CATACLYSMAL; Bursting; Catastrophic

CHAFFY; Trivial; Worthless

COMMENDABLE; Laudable; Praiseworthy

CRACKED; Flawed; Crazy 
CRUDE; Unpolished; Unprepared

CRUMMY; Cheap; Trashy

DAFT; Insane; Ridiculous

DAMNABLE; Detestable; Odious

DASTARDLY; Treacherously cowardly

DEMEANED; Debased in dignity or stature

DEPLORABLE; Lamentable; Calamitous

DISGRACEFUL; Deserving disgrace; Dishonorable

DISLUSTERED; Deprived of radiance or distinction

EVILDOER; Wrongdoer; Malefactor

EXCEPTIONAL; Remarkable; Extraordinary

FLAGRANT; Notorious; Scandalous

FUTILE; Frivolous; Trivial

GEM; Person held in great esteem or affection

GOOD; Pleasing; Satisfactory

GOOD-FOR-NOTHING; Useless; Worthless

HALF-WITTED; Foolish; Stupid

HALOED; Possessing quality of glory, majesty, sanctity

HONORABLE; Nobel; Illustrious

IGNOMINIOUS; Contemptible; Discreditable

LACKLUSTER; Lacking brilliance; Dull

LOVEWORTHY; Worthy of affection

MAGNIFICENT; Brilliant; Splendorous

MEANINGLESS; Purposeless, without meaning, significance or value

MENIAL; Servile; Slavish

MIGHT-HAVE-BEEN; A person who might have amounted to something

MORONIC; Stupid; Lacking good judgment

NEFARIOUS; Extremely wicked

NEGLIGIBLE; Insignificant; Trifling

NINNYISH; Fool; Simpleton

NOBLE; Honorable; Reputable

NONEXPERT; Not an expert

NOODLE; Stupid; Simpleton

NOVEL; Original or striking in conception or style

ODDISH; Strange; Queer

ONE-HORSE; Small and unimportant

ORDUROUS; Filthy; Obscene

OVERGREAT; Above and beyond great; Markedly superior

PEACHY; Sweet; Fair

PECULIAR; Strange; Unusual

PIDDLING; Trifling; Trivial

PRUNE; Simpleton; Fool

REMARKABLE; Extraordinary; Unprecedented

SCALAWAG; Scamp; Rascal

SCRUMPTIOUS; Delectable; Splendid to the senses

SCUMMY; Low class; Worthless

SENSATIONAL; Melodramatic; Emotional

STUPE; Stupid 
TOMFOOL; Foolish and silly

TOPGALLANT; Grand; The best

TOPNOTCH; First-rate; Unsurpassed

UNADMIRABLE; Censurable; Reproachable

UNCAPABLE; Incapable; Unable

UNCROOKED; Unbent; Straight

UNENVIABLE; Not desirable

UNFALLEN; Not falling back in performance

UNFLAWED; One who has no shortcomings

UNGIFTED; Not talented

UNMARRED; Not defaced; Not blemished

UNMERITORIOUS; Not deserving of praise

VIRTUOUS; Morally good

WARPED; Mentally twisted; Perverted

WRONG-HEARTED: Wrong or perverse in feeling; Unjust

\section{Set 3}

ABUNDANT; More than adequate

ANOMALOUS; Abnormal; Deviant

AUTHENTIC; Trustworthy; Credible

BARREN; Uninteresting; Dull

BASE; From low moral standards; Valueless

BASEBORN; Plebeian; Vile

BLEAR-WITTED; Dim-witted; Dull of mind

BRAINLESS; Witless; Mindless

BUNGLESOME; Inept; Inefficient

CATTLE; Class of contemptible human beings

CHARACTERLESS; Without individuality; Dependent

COCKBRAINED; Foolish; Scatterbrained

COMPETENT; Characterized by marked or sufficient aptitude, skill, strength, or knowledge

CONSEQUENTIAL; Pompous; Self-important

CURSED; Deserving to be condemned; Detestable

DAMNABLE; Detestable; Odious

DEMIGOD; One so prominent in intellect, power, ability, beneficence, or appearance as to seem to approach the divine

DERISIBLE; Worthy of derision or scorn

DISPARAGEABLE; Unworthy; Discredited

DISTINCTIVE; Unique; Singular

DOLTISH; Stupid; Foolish

DREGGY; Dirty; Repulsive

EXTRAORDINARY; Exceptional; Unusual

FAULTFUL; Full of imperfections and errors

FIRST-RATE; Excellent

FLAGRANT; Notorious; Scandalous

GOOSISH; Silly; Foolish

GRAND; Magnificent; Splendid 
GUMP; Foolish; Dull person

HONEY; Sweet; Beloved

INDISTINCTIVE; Without unique characteristics

INEXPERT; Inexperienced; Not skilled

INGLORIOUS; Disgraceful; Disreputable

INSUFFERABLE; Intolerable; Unbearable

LACKALL; A person who lacks everything

LAUREATE; Distinguished; Honored

LOUSY; Contemptible; Disgusting

MIDDLE-RATE; Mediocre

MISBESEEMING; Not appropriate

NONESSENTIAL; Not of prime or central importance

ODD; Strange; Eccentric

OFF; Away from what is considered normal, regular, standard

PARAGON; A model of excellence

PEACHY; Sweet; Fair

PILL; Tiresome; Disagreeable person

PITHSOME; Forceful; Strong

PROWESSFUL; Of exceptional valor; Bravery

REPREHENSIBLE; Deserving blame or censure

RESPECTABLE; Reputable; Estimable

ROTTER; Blackguard; Lazy person

SLAP-UP; First rate; Fine; Excellent

SMIRCHLESS; Clean; Without blemish

SNIPE; A fool

STAINED; Disreputable; Dishonoured

STUPE; Stupid

SUBNORMAL; Inferior; Defective

SWELL; First-rate; Grand

SWELLISH; Stylish; Fine

UNABLE; Impotent; Helpless

UNBONNY; Ugly, unhealthy

UNCAPABLE; Incapable; Unable

UNCONSEQUENTIAL; Inconsequential; Unimportant

UNDEBASED; Not reduced in quality or value; Not adulterated

UNDEPRAVED; Not corrupt

UNDESERVING; Not worthy of praise; Not meritorious

UNFLAWED; One who has no shortcomings

UNMARVELOUS; Not wonderful; Not of high quality

UNMOMENTOUS; Not significant; Unimportant

UNPARAGONED; Matchless; Not paralleled

UNREMARKABLE; Lacking distinction; Ordinary

UNSATISFACTORY; Inadequate; Disappointing

UNSTAINED; Unblemished; Pure

UNSUFFERABLE; Intolerable; Obnoxious

VIRTUOSO; Master; Expert in the technique of an art

WOODEN-HEADED; Blockhead; Stupid 